\title{
Optimization of Accelerated Destructive Degradation Testing of Cementitious Materials for Their Performances Qualification under Aggressive Environments: The Case of Carbonation
}

\author{
Weian Yan, ${ }^{1,2}$ David Bigaud (DD, ${ }^{1}$ Nadare Matoiri Chaibati, ${ }^{1,3}$ and Laurent Izoret $^{3}$ \\ ${ }^{1}$ Laboratoire Angevin de Recherche en Ingénierie des Systèmes, LARIS, EA 7315, UNIV Angers, SFR MathSTIC, \\ 62 Avenue Notre-Dame-du-Lac, 49000 Angers, France \\ ${ }^{2}$ College of Transportation and Logistics, East China Jiaotong University, Nanchang, Jiangxi 330013, China \\ ${ }^{3}$ A.T.I.L.H.-Association Technique de l'Industrie des Liants Hydrauliques, 7 Place de la Défense, 92974 Paris la Défense, France
}

Correspondence should be addressed to David Bigaud; david.bigaud@univ-angers.fr

Received 23 November 2019; Revised 7 February 2020; Accepted 10 March 2020; Published 9 April 2020

Academic Editor: Giovanni Lancioni

Copyright (c) 2020 Weian Yan et al. This is an open access article distributed under the Creative Commons Attribution License, which permits unrestricted use, distribution, and reproduction in any medium, provided the original work is properly cited.

In order to guarantee the performance or to qualify the risk of nonperformance of cementitious materials over time, a significant number of experimental data obtained from tests mimicking various degradation mechanisms are required. The slowness of the materials' degradation under environmental service conditions is an issue, and thus, acceleration strategies are required to obtain reliable and comprehensive results in a shorter time. The objective of this research work is to provide a generic framework for the design of optimal accelerated destructive degradation tests (ADDTs) for cementitious materials qualification. The definition of the optimal design of experiments depends on the capacity to capture the influence of data variability and uncertainty from any sources; they are extracted either from physical models or from experimental tests. In this research, the evolution of carbonation depth is characterized with the Wiener process formalism and the random effects related to the material heterogeneity are taken into account. Once the process parameters are estimated through the maximum likelihood estimation (MLE), associated with the expectation-maximization (EM) algorithm, we provide a step-by-step and detailed method to investigate the optimal design of ADDTs. The latter is defined as the one for which we can estimate durability indicators such as mean-time-to-failure with the best accuracy based on three criteria (D-V-A optimality) considering constraints of time, total number of samples, or limited costs. The optimal total sample size for the accelerated carbonation test and the optimal sample size allocation proportions for each stress level are determined, and the effects of the stress level on the objective functions and of test time duration constraint are also discussed. A comparison of the relative efficiency of optimal three-level versus optimal two-level ADDT completes this work.

\section{Introduction}

Production and, by extension, qualification of concrete are based on the EN 206 standard [1]. Considering 18 exposure classes, this standard prescribes the mix designs in order to guarantee a 50-year durability for buildings and 100-year durability for engineering structures. Construction actors have to consider new challenges such as dealing with environmental issues, seeking more resistant building materials, and reducing construction budget. Thus, this may lead to using concrete with a composition that differs from that recommended in EN 206. This alternative is acceptable if the new concrete is at least as good as the concrete defined in the standard, under the specific exposure class for which we want to qualify the performance of the new concrete. This approach called "performance-based" [2], based on the demonstration of the performance's equivalency, has gained large success in many fields [3]. This demonstration can be made through three different ways: by using durability indicators, performance tests [4], or a proven physical model. 
Among the durability indicators, general indicators, "general" because they are not related to a unique mechanism of degradation (e.g., water porosity and water permeability), are distinguished from specific indicators (e.g., carbon dioxide or chloride diffusion coefficients). The performance test allows assessing directly the degradation process within the concrete (e.g., carbonation depth evolution in order to qualify the concrete performance under the XC exposure class). The physical model allows modelling the degradation phenomenon on the concrete. In that case, concrete is qualified if the model proves that the concrete performs well for a given environment. In our work, we propose a mixed approach combining both a physical model and optimally designed performance tests.

For cementitious materials, the carbonation phenomenon evolves slowly over time under normal or service use. It takes time to observe damage on the concrete within a short test duration. In such a case, an accelerated degradation test (ADT) by elevating the stress level can be used to obtain the typical degradation process within an accurate test duration and then to extrapolate the product's lifetime information under normal operating conditions. Generally, most of the existing concrete durability tests are destructive but there are also nondestructive tests under development and investigation in order to resolve problems such as test duration and high cost $[5,6]$. In this paper, we focus only on the case of destructive carbonation tests-which are the most highly represented for carbonation tests-and we will talk hereinafter about optimization of accelerated destructive degradation tests (denoted by acronyms ADDT or A(D)DT if the proposed theoretical developments can be applied either on ADT or ADDT) which is less straightforward to deal with ADT (i.e., nondestructive tests).

A(D)DT has become an efficient approach to reliability assessment or lifetime prediction for degrading products [7]. Depending on the different stress loadings application, A(D) DTs can be classified into constant-stress A(D)DT (CSA(D) $\mathrm{DT})$, step-stress $\mathrm{A}(\mathrm{D}) \mathrm{DT}$ (SSA(D)DT), and progressivestress $A(D) D T$ (PSA(D)DT). In order to maximize their efficiency-to-cost ratio, we must carefully design the A(D) DT to obtain the lifetime information at the service condition. Therefore, the optimal design problem of an A(D)DT experiment received considerable attention from reliability researchers and engineers [8]. Among them, CSA(D)DT is the most popular method in practical applications. For example, Duan and Wang [9] and Tsaï et al. [10] address the optimal design problems for CSADT-based gamma process. Liu et al. [11] focus on Inverse Gaussian processes. Tang et al. [12] and Chen et al. [13] deal with the optimal design for degradation tests based on a nonlinear generalized Wiener process with random effects. In a CSA(D)DT, all test samples are divided into several groups, and each group of samples is exposed to a severe stress level. Under each stress level, the corresponding samples are inspected independently, and their degradation paths are recorded at the prespecified times. In the CSA(D)DT, the experienced stress level does not change for each sample, which requires more samples than for SSA(D)DT [14]. At the same time, the failure mechanisms affected by stress level do not change, which makes CSA(D)DT easier to deal with than SSA(D)DT. For the concrete, since samples are not expensive and can be easily produced, to choose CSA(D)DT plans rather than SSA(D)DT ones is definitely relevant.

This article provides a method for the design of optimal (with constrained sample sizes, time measures, and costs) CSADDT used to qualify concrete durability or reliability indicators under normal service conditions. Additionally, the data required for the implementation of our method can come from either performance tests carried out in a laboratory or using physical models, which can help to obtain prior data in the absence of available experimental data. Different types of physical models describing concrete performances exist. They can be divided into three main categories: empirical, semiempirical, and numerical. Among these models, for the specific case of carbonation process, which is the subject of the present article, we can mention Papadakis, Duracrete, Houst-Wittmann, Yang, and Hyvert models (all described in [15]). In our approach, a physical model is used to generate data mimicking the degradation process, while taking into account the uncertainties close to those observed in reality. The aim is to find a prior optimal design plan ADDT but not necessarily to have a model as fair as possible; the important point is that the physical models are merely used to generate prior data, which will be subsequently processed through degradation models whose formalisms are particularly suited for the design of optimal ADDT plan. Due to the limited availability of experimental data for carbonation tests, we went with the Hyvert carbonation model in order to simulate data tests. This model takes into account the change of $\mathrm{CO}_{2}$ pressure (which will be the acceleration stress) applied on concrete, and most of its input parameters have known statistical distribution laws that allow simulating uncertainty of degradation data (carbonation depth over time).

There are two main formalisms of degradation models: stochastic process models and general path models. The latter models are very easy to use; the theory has been well established and is more robust than process-based models. Lu and Meeker [16] and Pettit and Young [17] provide a general discussion about this approach. The main issue in performance tests is that the measured characteristics are random variables. This is due to measurement errors, to the inherent variability of concrete properties, and finally to the environmental conditions (temperature, humidity, $\mathrm{CO}_{2}$, etc.) which are random and time dependent. From this view, both stochastic process models and general path ones can effectively characterize the uncertainty of the degradation process $[18,19]$. For our generic approach, we use a physical model to get prior alternative data with a degradation dynamic following a square root function of time (see Section 2.1 and equation (1)). It motivates the choice of stochastic models which are more suitable for the consideration of physical mechanisms.

In a study about the carbonation behavior of concrete made of recycled aggregates, Zhang and Xiao [20] have represented the evolution of degradation in the concrete by a stochastic process. The evolution over time of the carbonation depth is followed. This degradation process is 
monotonic, and they have chosen specifically the Gamma process for its suitability to model progressive and monotonic increasing damage. Even if it is not formally monotonic, the Wiener process can also be used to model the evolution of damage because it shows increasing degradation on average and that the possible negative increment of degradation modelled for an infinitesimal time interval can be hold physically accountable to measurements errors or to the variability between two tested samples. For the latter case indeed, as the carbonation tests are destructive, there is no guarantee to observe a monotonic evolution of degradation between two test times since the measurements are not made on the same sample (they are destroyed during the test). Thus, this stochastic degradation process is very flexible; it not only can be used to model monotonic degradation processes but also can be used to model nonmonotonic degradation processes. In this regard, the Wiener process is suitable for the carbonation process. The Wiener process has also many nice properties; e.g., the random effects and explanatory variables can be flexibly incorporated into the model. If the distribution of the measured characteristic is not normal, an "isoprobabilistic transformation," which consists in transforming the initial distribution law in an equivalent normal one (with, for example, the same entropy and median, or, the same median and a quartile, depending on the properties we want to retain) can still be applied to enforce the data to be pseudonormal.

To define precisely its objectives, as already mentioned, our research work focuses on the development of a "generic" method for the prescription of optimal CSADDT used to qualify concrete durability. This method is called "generic" since it can be applied for any degradation mechanisms. It can exploit data either from experimental preliminary tests or from proven physical models and can deal with any kind of random degradation data with the nonlinear generalized Wiener process. To demonstrate the relevancy of our method, we apply it to the case of the degradation of the concrete by carbonation. This is one of the most studied degradation phenomena on the concrete. Concrete carbonation leads to the corrosion of reinforced concrete and cause damages that destroy the structures. Thus, civil engineers conduct many studies in the field of accelerated carbonation tests, carbonation diffusion, and models in order to understand this phenomenon and to predict the lifetime of reinforced concrete structures [21].
The remainder of the paper is arranged as follows. Section 2 will present the physical and stochastic carbonation process modelling. Hyvert model, used in the first stage of data generation, will be detailed before the presentation of the nonlinear generalized Wiener process. The second stage of our generic method consists in the maximum likelihood estimations (MLEs) of parameters completed by the Expectation-Maximization (EM) algorithm (Section 3.1). The third stage of durability estimation is developed in Section 3.2. Section 4 describes the fourth stage of the generic method: how to design optimal CSADDT for the destructive test. The proportion of units allocated to each stress level and test stress levels will be determined based on three optimization criteria. In subsequent Section 5, we propose to apply our method to the case of the qualification of concrete durability under carbonation, with the aim to show the performance of the proposed methods. Section 6 concludes the paper.

A graphical abstract is proposed to assist in the understanding of our approach (Figure 1).

\section{Carbonation Process Modelling}

As illustrated in Figure 1, the very first stage of our approach is to collect data from degradation tests or from physical laws and to model these data using a stochastic process formalism suitable for the optimization of ADDT.

2.1. Probabilized Physical Model. As already mentioned, availability of comprehensive experimental carbonation tests data is limited. Use of a carbonation physical model can be an option to get prior alternative data (stage 1 of the graphical abstract). In the following, we have used the Hyvert carbonation model in order to simulate data tests. This model provides the value of the carbonation depth $Y_{\text {data }}$ as a function of time $t$. It explicitly involves the influence of the $\mathrm{CO}_{2}$ pressure at the concrete surface $P_{0}$, which will be the acceleration stress. The reason of the choice of the Hyvert model is not only based on a criterion of accuracy, but it relies more specifically on the fact that its main input parameters have known statistical distribution laws that allow simulating uncertainty effect on the simulation of carbonation depth value $Y_{\text {data }}(t)$. This carbonation depth can be estimated as

$$
Y_{\text {data }}(t)=\sqrt{\frac{2 . \operatorname{err} 1\left(\left(D_{\mathrm{CO} 2}^{o} / Q 1\right)_{\mathrm{ref}}\right) \cdot Q 1 \cdot P_{0} \cdot t \cdot k_{e} \cdot k_{p}}{\text { R.T. }\left(1+23.5 C 2^{\prime} \cdot\left(P_{0} / P_{\mathrm{atm}}\right)^{0.67}\right)\left(C 2^{\prime} / 0.67+1 \cdot\left(P_{0} / P_{\mathrm{atm}}\right)^{0.67}+\mathrm{Q} 1\right)}},
$$

with $\left(D_{\mathrm{CO} 2}^{o} / \mathrm{Q} 1\right)_{\mathrm{ref}}=6.44 \cdot 10^{-13} \cdot\left(36.4 \cdot e^{-0.04 f_{\mathrm{cm}, 28}}\right)^{2}$ when $D_{\mathrm{CO} 2}^{o}$ is not available.

The meanings of the model parameters, their characteristic values, and distribution statistical laws, if available, used for the prior database generation are reported in Table 1.
2.2. Degradation Processes. The key point of the first stage consists in modelling data through a formalism suitable for optimization. Stochastic processes, with their capacity to characterize the uncertainty and dynamics of the degradation process, have been used for this purpose. Stochastic processes are increasingly used by engineers to predict the 


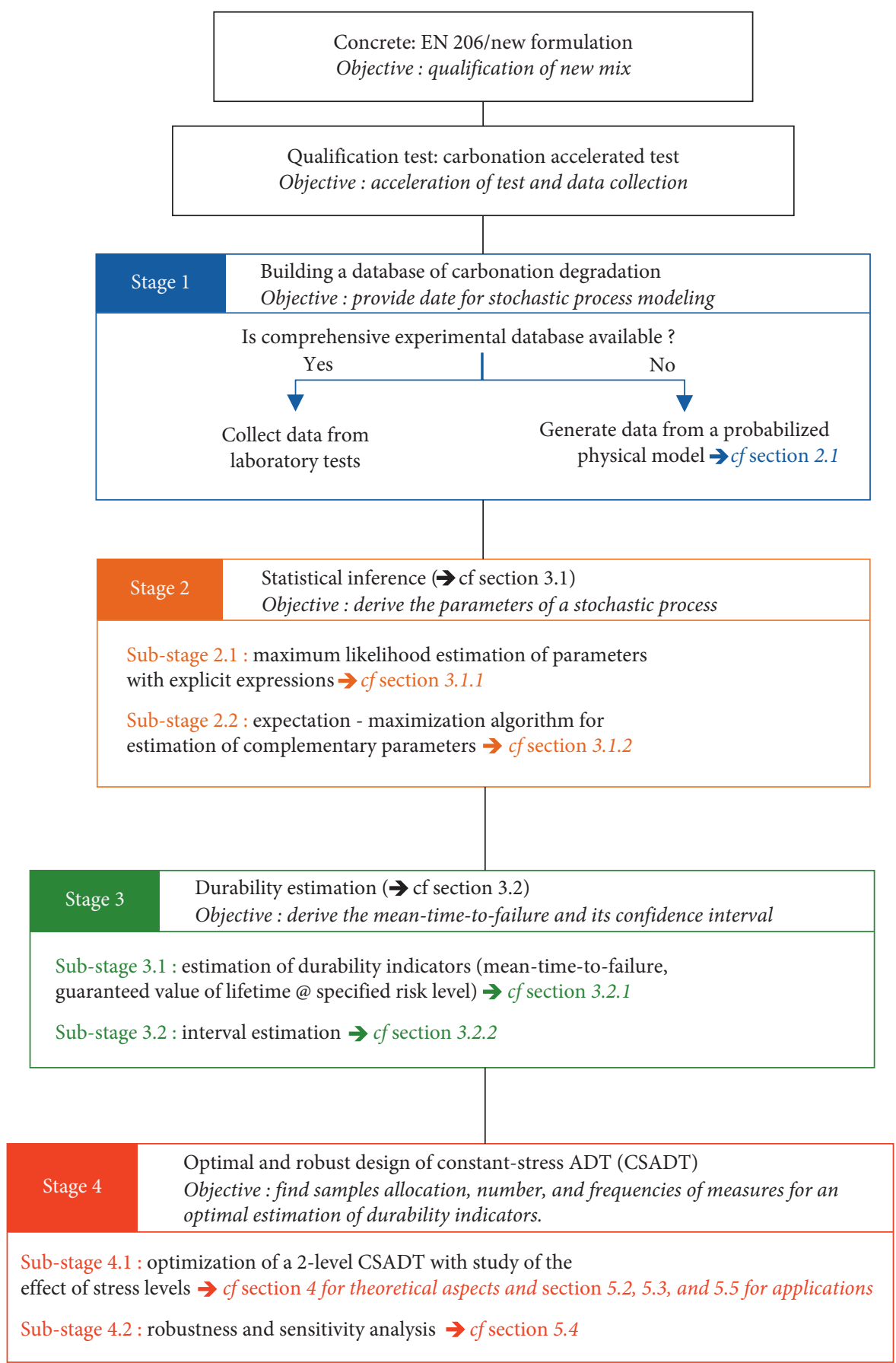

FIGURE 1: Graphical abstract to illustrate the optimization of an accelerated carbonation test of cementitious materials.

reliability of products. There are different types of stochastic models in the literature. Among them, the Wiener process has received widespread attention in degradation data analysis and performs well. The primary reason why we select the Wiener process to model the carbonation depth evolution is that from the physical standpoint, the degradation phenomena can be viewed as an accumulation of additive and irreversible damages caused by a sequence of internal and external random shocks. Wiener process is very suitable to model this.
We propose here to analyze the degradation by using a nonlinear generalized Wiener process, which can be expressed as

$$
Y_{\text {mod }}(t)=\eta \Lambda(t)+\sigma_{B} B(\Lambda(t))
$$

where $\eta$ is the degradation rate, which represents the speed of product change from normal to failure. Generally speaking, the greater the stress condition, the larger the $\eta$. $\Lambda(t)$ is the function of time, which is specified according to degradation physics or empirical observations. For the 
TABLE 1: Input parameters for the Hyvert model to generate data (from [15]).

\begin{tabular}{|c|c|c|c|c|c|}
\hline Parameters & Units & Description & $\begin{array}{c}\text { Characteristic } \\
\text { values } \\
\text { (mean-variance) }\end{array}$ & $\begin{array}{l}\text { Standard } \\
\text { deviation }\end{array}$ & $\begin{array}{c}\text { Statistic } \\
\text { distribution }\end{array}$ \\
\hline err1 & - & Error estimation of $\left(D_{\mathrm{CO}_{2}}^{o} / Q 1\right)_{\mathrm{ref}}$ & 0.84 & 0.52 & Lognormal \\
\hline $\mathrm{D}_{\mathrm{CO}_{2}}^{0}$ & $\mathrm{~m}^{2} / \mathrm{S}$ & Diffusion coefficient out of porous zone & $114.10^{-7}$ & $182.10^{-8}$ & Normal \\
\hline Q1 & $\mathrm{Mol} / \mathrm{l}$ & Quantity of Portlandite $\mathrm{Ca}(\mathrm{OH})_{2}$ & 2.19 & - & Deterministic \\
\hline$f_{\mathrm{cm}, 28}$ & $\mathrm{MPa}$ & Compressive strength of the concrete & 35 & 2 & Lognormal \\
\hline$P_{0}$ & $\mathrm{~Pa}$ & $\mathrm{CO}_{2}$ pressure on the concrete surface & 30 & - & Deterministic \\
\hline$K_{e}$ & - & $\begin{array}{c}\text { Parameter taking into account environmental } \\
\text { conditions }\end{array}$ & 1 & 0.1 & Normal \\
\hline$K_{p}$ & - & Parameter taking into account thermal treatment & 0.49 & - & Deterministic \\
\hline$P_{\mathrm{atm}}^{P}$ & $\mathrm{~Pa}$ & The atmospheric pressure & $10^{5}$ & - & Deterministic \\
\hline$T$ & $\mathrm{~K}$ & The temperature in Kelvin & 293 & - & Deterministic \\
\hline $\mathrm{R}$ & $\mathrm{J} / \mathrm{K} \cdot \mathrm{mol}$ & The perfect gases constant & 8.31 & - & Deterministic \\
\hline$C 2^{\prime}$ & $\mathrm{mol} / \mathrm{l}$ & The quantity of CSH-hydrated calcium silicate & 2.34 & - & Deterministic \\
\hline
\end{tabular}

concrete, the mechanism of concrete carbonation is studied by several well-established models $[22,23]$ which demonstrates that $\Lambda(t)=\sqrt{t}$.

According to the definition of Wiener process, $Y_{\text {mod }}(\Lambda(t))$ may decrease between two consecutive times while, in fact, degradation process is constantly increasing and monotonic. But, as we explain in the introduction, the Wiener process can be applied to model the evolution of damage because it shows increasing degradation on average and that the possible decrease between two times can be used to take into account the measurement errors and the variability between two tested samples. Moreover, when $\eta \Lambda(t)$ is "large" compared with $\sigma_{B} \sqrt{\Lambda(t)}$, e.g., $\eta \Lambda(t) \gg \sigma_{B} \sqrt{\Lambda(t)}$; therefore, the probability that degradation increment is negative becomes small and can be ignored. This is the case in our situation. This tendency is enhanced when $\Lambda(t)$ increases over time [24]. Therefore, Wiener process can be used to model the degradation path whether the degradation process is monotonous or not.

2.3. Accelerated Carbonation Test. The accelerated stress is the factor that accelerates the studied degradation phenomenon. In our case, the stress used is the $\mathrm{CO}_{2}$ concentration $P_{0}$ and it is kept constant to be compliant with the different standards on accelerated carbonation tests. The optimization work will consist, among other parameters to adjust, in determining the number of stress levels and samples for each level in order to provide a better estimates' accuracy under constraints of total sample size, total duration of tests, or total costs.

In the model (equation (2)), $\eta$ denotes the degradation rate which obviously should change when the acceleration stress is different. The link function between the degradation rate and stress level can follow one of the three functions as follows:
(i) Power law relation: $\eta\left(S_{k}\right)=\xi_{0} S_{k}^{\vartheta}$
(ii) Arrhenius relation: $\eta\left(S_{k}\right)=\xi_{0} e^{-\vartheta / S_{k}}$
(iii) Exponential relation: $\eta\left(S_{k}\right)=\xi_{0} e^{9 S_{k}}$

Here, $\xi_{0}$ and $\vartheta$ are the constants to be determined and $S_{k}$ is the $k$ th level of stress, stress considered here to be the $\mathrm{CO}_{2}$ pressure $P_{0}$.

Prior standardization of $S_{k}$ can be applied to obtain a unique form of the degradation rate:

$$
\eta\left(s_{k}\right)=\alpha e^{\beta s_{k}},
$$

where $s_{k}$ represents the $k$ th standardized stress level, which is defined as

$$
s_{k}= \begin{cases}\frac{\ln S_{k}-\ln S_{0}}{\ln S_{\max }-\ln S_{0}}, & \text { for the power law relation, } \\ \frac{\left(1 / S_{k}\right)-\left(1 / S_{0}\right)}{\left(1 / S_{\max }\right)-\left(1 / S_{0}\right)}, & \text { for the Arrhenius relation, } \\ \frac{S_{k}-S_{0}}{S_{\max }-S_{0}}, & \text { for the exponential relation, }\end{cases}
$$

where $S_{0}$ and $S_{\max }$, respectively, are the normal or service stress and the maximum physically allowable stress, respectively. Under the standardization, $s_{0}=0<s_{1}<\cdots<s_{k}<\cdots<s_{\max }=1$.

For the concrete, due to design tolerances, manufacturing variation, and other uncertainties, reliability of the same type of concrete may have inherent difference called heterogeneity. In order to get a more accurate reliability assessment result, it is necessary to incorporate the heterogeneity into the degradation assessment model. This measure has a theoretical value and practical engineering significance, which has been confirmed by many studies $[16,25]$. In our case, the parameter $\alpha$ is assumed to follow normal distribution and it is s-independent from stress levels. After the transformation, the model can be expressed as

$$
\left\{\begin{array}{l}
Y_{\text {mod }}(t)=\alpha e^{\beta s_{k}} \Lambda(t)+\sigma_{B} B(\Lambda(t)), \\
\alpha \sim N\left(\mu_{\alpha}, \sigma_{\alpha}^{2}\right), \Lambda(t)=\sqrt{t},
\end{array}\right.
$$


where the unknown parameters of the process are $\Theta=\left(\mu_{\alpha}, \sigma_{\alpha}^{2}, \beta, \sigma_{B}^{2}\right)$.

Once the parameters are determined, the Wiener process can be used, for example, to find the statistical distribution of the carbonation depth for a set value of time, or of the duration for an allowable carbonation depth (see Sections 3.2 and 4.1, for the latter). But its main use will be to design an optimal ADDT (see Sections 4 and 5).

2.4. Notations and Assumptions. To use Wiener process for the purposes mentioned above, initial choices and assumptions have to be put forward:

(1) We will use CSADDT for its operational convenience. The number of stress levels is denoted by " $d$," and, thus, $s_{k}, k=1,2, \ldots, d$. The test is destructive, i.e., a unit can only be tested once.

(2) The total number of units available for the test is $N$; $N_{k}$ of them are allocated to the stress level $s_{k}$, such that $\sum_{k=1}^{d} N_{k}=N$. Using a different unit allocation at all levels of an accelerating variable is recommended by Meeker et al. [8].

(3) Let $m_{k}$ and $f_{k}$, respectively, represent the number and frequency of measurements for units at the stress level $s_{k}$. Transformed time is $\Lambda\left(t_{k j}\right)$, such that $\Lambda\left(t_{k j}\right)=\sqrt{j f_{k}}$. And the corresponding number of samples is $n_{k j}$, such that $\sum_{j=1}^{m_{k}} n_{k j}=N_{k}$. We assume the same number of samples at each test time $n_{k 1}=n_{k 2}=, \ldots,=n_{k m_{k}}=n_{k}$, such that $m_{k} n_{k}=N_{k}$. And set $p_{k j}=\left(n_{k j} / N\right)=p_{k}, j=1,2, \ldots, m_{k}$, such that $\sum_{k=1}^{d} \sum_{j=1}^{m_{k}} p_{k j}=\sum_{k=1}^{d} p_{k} m_{k}=1$.

(4) Under each stress level $s_{k}, k=1, \ldots, d$, the degradation characteristic $y_{k j i}$ of the ith unit $\left(i=1, \ldots, n_{k}\right)$ follows normal distribution with mean $\alpha_{k j i} i^{\beta s_{k}} \Lambda\left(t_{k j}\right)$ and variance $\sigma_{B}^{2} \Lambda\left(t_{k j}\right)$ given that the value of $\alpha$ is $\alpha_{k j i}$. Since the test is destructive and the initial degradation measurement is 0 , then degradation increment $\Delta y_{k j i}=y_{k j i}$.

(5) A unit is assumed to fail at time $t^{*}$ when its degradation $Y_{\text {mod }}\left(t^{*}\right)$ crosses a predetermined failure threshold $\omega$.

\section{Process Parameters and Durability Estimation}

\subsection{Parameter Estimation}

3.1.1. Maximum Likelihood Estimation (MLE). The MLE corresponds to substage 2.1 in the graphical abstract. It aims in deriving process parameters with explicit expressions.

The unknown parameters of the nonlinear generalized Wiener process have to be estimated from data provided by actual (experiments) or simulated (Hyvert model) accelerated carbonation tests. Following the argument in the assumptions, given the value of $\alpha_{k j i}$, the $\operatorname{PDF} f\left(y_{k j i} \mid \alpha_{k j i}\right)$ of degradation data $y_{k j i}$ is normally distributed with mean $\alpha_{k j i} e^{\beta s_{k}} \Lambda\left(t_{k j}\right)$ and variance $\sigma_{B}^{2} \Lambda\left(t_{k j}\right)$. Therefore, the likelihood function of the proposed model is

$$
L=\prod_{k=1}^{d} \prod_{j=1}^{m_{k}} \prod_{i=1}^{n_{k j}} \int_{-\infty}^{\infty} f\left(y_{k j i} \mid \alpha_{k j i}\right) \times f\left(\alpha_{k j i}\right) \mathrm{d} \alpha_{k j i} .
$$

is

Then, the log-likelihood function of $\Theta$, up to a constant,

$$
l=\ln L=-\frac{1}{2} \sum_{k=1}^{d} \sum_{j=1}^{m_{k}} \sum_{i=1}^{n_{k j}}\left(\frac{B_{2 k j i}^{2}}{\Lambda\left(t_{k j}\right) B_{1 k j}}+\ln \Lambda\left(t_{k j}\right)+\ln B_{1 k j}\right),
$$

where $B_{1 k j}=\sigma_{B}^{2}+\sigma_{\alpha}^{2} e^{2 \beta s_{k}} \Lambda\left(t_{k j}\right)$ and $B_{2 k j i}=y_{k j i}-e^{\beta s_{k}} \mu_{\alpha}$ $\Lambda\left(t_{k j}\right)$.

In the particular case of partially CSADDT (where the lower stress level used is the nominal standardized condition $s_{0}$ ), the log-likelihood function can be written:

$$
\begin{aligned}
l= & \ln L=-\frac{1}{2}\left(\frac{B_{\left(2 j i / s_{0}\right)}^{2}}{\Lambda\left(t_{\left(j / s_{0}\right)}\right)}+\ln \Lambda\left(t_{\left(j / s_{0}\right)}\right)+\ln B_{\left(1 j / s_{0}\right)}\right) \\
& -\frac{1}{2} \sum_{k=1}^{d} \sum_{j=1}^{m_{k}} \sum_{i=1}^{n_{k j}}\left(\frac{B_{2 k j i}^{2}}{\Lambda\left(t_{k j}\right) B_{1 k j}}+\ln \Lambda\left(t_{k j}\right)+\ln B_{1 k j}\right) .
\end{aligned}
$$

where $t_{\left(j / s_{0}\right)}$ corresponds to the specific time sequence of tests at nominal standardized conditions $s_{0}, y_{\left(j i / s_{0}\right)}$ are the associated $i$ degradation data obtained at $t_{\left(j / s_{0}\right)}$ under $s_{0}$, $B_{1 j / s_{0}}=\sigma_{B}^{2}+\sigma_{\alpha}^{2} \Lambda\left(t_{\left(j / s_{0}\right)}\right)$, and $B_{\left(2 j i / s_{0}\right)}=y_{\left(j i / s_{0}\right)}-\mu_{\alpha} \Lambda\left(t_{\left(j / s_{0}\right)}\right)$.

The MLEs of unknown parameters $\Theta$ can be obtained by maximizing log-likelihood function (7). But, generally speaking, there are no explicit expressions for these unknown parameters. Although the MLEs can be obtained numerically, the log-likelihood function is rather sensitive to parameter $\beta$; thus, the estimates can substantially fluctuate. At the same time, according to the recommendations given by Mc Lachlan and Krishnan [26], the EM algorithm can effectively provide the estimates of parameters and offer a simpler framework for computation of the MLEs. Therefore, the EM algorithm is adopted to obtain the MLEs of unknown parameters.

3.1.2. EM Algorithm. This section corresponds to substage 2.2 of the generic approach illustrated in the graphical abstract. The EM algorithm is an iterative algorithm, which includes the expectation step (E-Step) and the maximization step (M-Step). The E-step gives the Q-function by taking the expectation of complete-data log-likelihood function. The M-step maximizes the Q-function to update the parameter estimates, which often have a simple closed form. The EM algorithm is efficient in finding the MLEs when computation of the expectation and the maximization is easy to perform [26].

The joint PDF of $y_{k j i}$ and $\alpha_{k j i}$ is $f\left(y_{k j i}, \alpha_{k j i}\right)=$ $f\left(y_{k j i} \mid \alpha_{k j i}\right) \cdot f\left(\alpha_{k j i}\right)$. Then, the conditional distribution of $\alpha_{k j i}$ can be obtained by integrating $y_{k j i}$ out of the joint PDF, which yields $f\left(\alpha_{k j i} \mid y_{k j i}\right)$ that follows normal distribution with mean $\mu_{k j i y} \triangleq\left(\mu_{\alpha} \sigma_{B}^{2}+\sigma_{\alpha}^{2} e^{\beta s_{k}} y_{k j i}\right) /\left(\sigma_{B}^{2}+\sigma_{\alpha}^{2} e^{2 \beta s_{k}} \Lambda\left(t_{k j}\right)\right)$ 
and variance $\sigma_{k j i y}^{2} \triangleq\left(\sigma_{\alpha}^{2} \sigma_{B}^{2}\right) /\left(\sigma_{B}^{2}+\sigma_{\alpha}^{2} e^{2 \beta s_{k}} \Lambda\left(t_{k j}\right)\right)$. Therefore, $E\left(\alpha_{k j i} \mid y_{k j i}\right)=\mu_{k j i y}$ and $E\left(\alpha_{k j i}^{2} \mid y_{k j i}\right)=\mu_{k j i y}^{2}+\sigma_{k j i y}^{2}$ which can be used to calculate the $Q$-function at the E-step of the EM algorithm.

Based on the observed degradation data, as well as the random effect $\alpha_{k j i}$, the complete-data log-likelihood function, up to a constant, is

$$
\begin{aligned}
l_{C}= & -\frac{1}{2} \sum_{k=1}^{d} \sum_{j=1}^{m_{k}} \sum_{i=1}^{n_{k j}}\left[\ln \sigma_{B}^{2}+\ln \Lambda\left(t_{k j}\right)+\frac{\left(y_{k j i}-\alpha_{k j i} e^{\beta s_{k}} \Lambda\left(t_{k j}\right)\right)^{2}}{\sigma_{B}^{2} \Lambda\left(t_{k j}\right)}\right. \\
& \left.+\ln \sigma_{\alpha}^{2}+\frac{\left(\alpha_{k j i}-\mu_{\alpha}\right)^{2}}{\sigma_{\alpha}^{2}}\right] .
\end{aligned}
$$

In the following, the EM algorithm is used to compute the estimator of the model parameter vector:

E-step: calculate the $Q$-function, which is the expectation of $l_{C}$. Assume that the current value of the model parameter vector is $\Theta^{(h)}=\left(\mu_{\alpha}^{(h)}, \sigma_{\alpha}^{2(h)}, \beta^{(h)}, \sigma_{B}^{2(h)}\right)$; then, the $Q$-function is

$$
\begin{aligned}
& Q\left(\Theta \mid \Theta^{(h)}\right)=-\frac{1}{2}\left[N \ln \sigma_{B}^{2}+\sum_{k=1}^{d} \sum_{j=1}^{m_{k}} n_{k j} \ln \Lambda\left(t_{k j}\right)+\sum_{k=1}^{d} \sum_{j=1}^{m_{k}} \sum_{i=1}^{n_{k j}} \frac{y_{k j i}^{2}}{\sigma_{B}^{2} \Lambda\left(t_{k j}\right)}\right. \\
& +\sum_{k=1}^{d} \sum_{j=1}^{m_{k}} \sum_{i=1}^{n_{k j}} \frac{e^{2 \beta s_{k}} \Lambda\left(t_{k j}\right) E\left(\alpha_{k j i}^{2} \mid y_{k j i}\right)}{\sigma_{B}^{2}}-\sum_{k=1}^{d} \sum_{j=1}^{m_{k}} \sum_{i=1}^{n_{k j}} \frac{2 y_{k j i} e^{\beta s_{k}} E\left(\alpha_{k j i} \mid y_{k j i}\right)}{\sigma_{B}^{2}} \\
& \left.+N \ln \sigma_{\alpha}^{2}+\sum_{k=1}^{d} \sum_{j=1}^{m_{k}} \sum_{i=1}^{n_{k j}} \frac{E\left(\alpha_{k j i}^{2} \mid y_{k j i}\right)}{\sigma_{\alpha}^{2}}-\frac{2 \mu_{\alpha}}{\sigma_{\alpha}^{2}} \sum_{k=1}^{d} \sum_{j=1}^{m_{k}} \sum_{i=1}^{n_{k j}} E\left(\alpha_{k j i} \mid y_{k j i}\right)+\frac{N \mu_{\alpha}^{2}}{\sigma_{\alpha}^{2}}\right] . \\
& \hat{\beta}_{M}=\frac{1}{d} \sum_{k=1}^{d} \frac{1}{s_{k}}\left[\ln \left(\sum_{j=1}^{m_{k}} \sum_{i=1}^{n_{k j}} y_{k j i} E\left(\alpha_{k j i} \mid y_{k j i}\right)\right)\right. \\
& \text { from the following system of equations: } \\
& \text { the first derivative of } \left.Q\left(\Theta \mid \Theta{ }^{(h)}\right) \text { with } \quad-\ln \left(\sum_{j=1}^{m_{k}} \sum_{i=1}^{n_{k j}} \Lambda\left(t_{k j}\right) E\left(\alpha_{k j i}^{2} \mid y_{k j i}\right)\right)\right]
\end{aligned}
$$

M-step: compute the first derivative of $Q\left(\Theta \mid \Theta^{(h)}\right)$ with respect to $\Theta$, and set its value to be zero. Then, $\Theta^{(h+1)}$ can be derived from the following system of equations:

$$
\begin{aligned}
& \widehat{\mu}_{\alpha}^{(h+1)}= \frac{1}{N} \sum_{k=1}^{d} \sum_{j=1}^{m_{k}} \sum_{i=1}^{n_{k j}} E\left(\alpha_{k j i} \mid y_{k j i}\right), \\
& \hat{\sigma}_{\alpha}^{2(h+1)}= \frac{1}{N} \sum_{k=1}^{d} \sum_{j=1}^{m_{k}} \sum_{i=1}^{n_{k j}} E\left(\alpha_{k j i}^{2} \mid y_{k j i}\right)-\left(\widehat{\mu}_{\alpha}^{(h+1)}\right)^{2}, \\
& \sum_{k=1}^{d} s_{k} e^{\hat{\beta}^{(h+1)}} s_{k}\left(A_{k 1}-e^{\hat{\beta}^{(h+1)}} s_{k} A_{k 2}\right)=0, \\
& \widehat{\sigma}_{B}^{2(h+1)}= \frac{1}{N} \sum_{k=1}^{d} \sum_{j=1}^{m_{k}} \sum_{i=1}^{n_{k j}}\left[\frac{y_{k j i}^{2}}{\Lambda\left(t_{k j}\right)}-2 y_{k j i} e^{\beta}{ }^{(h+1)} s_{k} E\left(\alpha_{k j i} \mid y_{k j i}\right)\right. \\
&+\Lambda\left(t_{k j}\right) e^{2 \hat{\beta}^{(h+1)}} s_{k} E\left(\alpha_{k j i}^{2} \mid y_{k j i}\right),
\end{aligned}
$$

where $A_{k 1}=\sum_{j=1}^{m_{k}} \sum_{i=1}^{n_{k j}} y_{k j i} E\left(\alpha_{k j i} \mid y_{k j i}\right)$ and $A_{k 2}=\sum_{j=1}^{m_{k}} \sum_{i=1}^{n_{k j}}$ $\Lambda\left(t_{k j}\right) E\left(\alpha_{k j i}^{2} \mid y_{k j i}\right), k=1,2$.

The parameter $\beta$ can be obtained according to the MATLAB function "fzero" or be approximately estimated as
It is worth noting that equation (12) is a one-dimensional equation-solved problem. Hence, it is easier than the direct solving of the likelihood function in equation (7).

To sum up and detail substage 2.2 of our generic method, the solution process of the EM algorithm is as follows (Algorithm 1):

\subsection{Durability Estimation}

3.2.1. Estimation of MTTF. The third stage of our generic method consists in the estimation of durability indicators from the stochastic process modelling.

To guarantee the durability of the concrete, it is important to know the failure time (substage 3.1 in the graphical abstract). The concept of first passage time (FPT) is often used to get the failure time. The durability of the concrete can be defined as the first time at which the carbonation reaches the steel rebars depth $\omega$, a critical value representing the distance between the concrete surface and 
Step 1: set the initial value of the parameters $\left(\mu_{\alpha}^{(0)}, \sigma_{\alpha}^{(0)}, \beta^{(0)}, \sigma_{B}^{2(0)}\right)$ and number of iterations $N_{\text {iter }}$ Step 2: obtain $\widehat{\mu}_{\alpha}^{(0)}$ according to equation (10)

Step 3: put $\widehat{\mu}_{\alpha}^{(h+1)}$ into equation (11) to obtain $\widehat{\sigma}_{\alpha}^{2(h+1)}$

Step 4: $\widehat{\beta}^{(h+1)}$ can be obtained by solving equation (12), or can be approximated by using (14)

Step 5: put $\widehat{\beta}^{(h+1)}$ into equation (13) to obtain $\widehat{\sigma}_{B}^{2(h+1)}$

Step 6: repeat $N_{\text {iter }}$ time from steps 2 and 5

Algorithm 1: Solution process of the EM algorithm.

the reinforcements. The first passage time represents the durability $(T)$ of the concrete and is defined by

$$
T=\inf \left\{t \mid Y_{\text {mod }}(t)>\omega\right\} \text {. }
$$

The durability $T$ conditioning on $\alpha$ under a stress level $s_{k}$ follows a transformation-inverse Gaussian distribution. Considering random effects in accelerated model (4), that is, $\alpha \sim N\left(\mu_{\alpha}, \sigma_{\alpha}^{2}\right)$, the PDF of $T$ by integrating $\alpha$ out of transformation-inverse Gaussian distribution becomes

$$
f_{T}(t)=\frac{\omega}{\sqrt{2 \pi\left(e^{2 \beta s_{k}} \Lambda^{4}(t) \sigma_{\alpha}^{2}+\Lambda^{3}(t) \sigma_{B}^{2}\right)}} \exp \left\{-\frac{\left(\omega-\mu_{\alpha} e^{\beta s_{k}} \Lambda(t)\right)^{2}}{2\left(e^{2 \beta s_{k}} \Lambda^{2}(t) \sigma_{\alpha}^{2}+\Lambda(t) \sigma_{B}^{2}\right)}\right\} .
$$

Then, the durability of the concrete under normal conditions of carbonation is

$$
T_{\mathrm{MTTF}} \approx \frac{2 \omega^{2}}{\sigma_{\alpha}^{2}}\left[D\left(\frac{\mu_{\alpha}}{\sqrt{2} \sigma_{\alpha}}\right)\right]^{2}
$$

where $D(z)=\exp \left(-z^{2}\right) \int_{0}^{z} \exp \left(u^{2}\right) \mathrm{d} u$ is the Dawson integral for all real $z$. According to the approximate property of Dawson integral, for large $z, D(z) \approx(1 /(2 z))$. Therefore, when $\left(\mu_{\alpha} / \sqrt{2} \sigma_{\alpha}\right)$ is large enough, $T_{\text {MTTF }}$ can be approximated as $T_{\text {MTTF }} \approx\left(\omega^{2} / \mu_{\alpha}^{2}\right)$.

The MLE $T_{\text {MTTF,M }}$ of the durability for the concrete can be obtained by substituting estimated parameters $\widehat{\Theta}_{M}$ into equation (17).

3.2.2. Interval Estimation. Once the MLE is carried out, the confidence interval of $T_{\text {MTTF }}$ can be obtained using its asymptotic normality. Unfortunately, it is not easy to carry out due to the complexity of Fisher information matrix. A more attractive alternative is to use the bootstrap method [27].

To sum up substage 3.2 of our generic method, the detailed procedure of the percentile bootstrap (PB) method and the bias-corrected percentile bootstrap (BCPB) method are outlined below in seven steps:
Step 1: generate $n$ samples $\alpha_{1}, \alpha_{2}, \ldots, \alpha_{n}$ from normal distribution $N\left(\widehat{\mu}_{\alpha}, \widehat{\sigma}_{\alpha}^{2}\right)$.

Step 2: substitute $\alpha_{i}$ into the model (2), and use the property of independent increments to generate $n$ simulated degradation paths.

Step 3: use the $n$ simulated degradation paths to estimate the parameters of model (5). Denote the bootstrap estimates of parameters as $\widehat{\Theta}_{B}$ (where " $B$ " index corresponds to the current Bootstrap repetition).

Step 4: substitute the bootstrap estimates $\widehat{\Theta}_{B}$ into (8) and then obtain the bootstrap estimate of durability $\widehat{T}_{\text {MTTF }, B}=T_{\text {MTTF }}\left(\widehat{\Theta}_{B}\right)$.

Step 5: repeat $B$ ( $B$ is a large number, e.g. $B=3000$ times from step 1 to step 4 )

Step 6: sort $\widehat{T}_{\mathrm{MTTF}, B}$ from small to large, and denote as $\widehat{T}_{\text {MTTF, },(1)}, \widehat{T}_{\text {MTTF, },(2)}, \ldots, \widehat{T}_{\text {MTTF, },(B)}$.

Step 7: the confidence interval of approximate 100 (1$\zeta) \%$ for the PB method and the BCPB method are, respectively, expressed as

$$
\begin{cases}\text { PB } & {\left[\widehat{T}_{\mathrm{MTTF}, B, \mathrm{LPB}}, \widehat{T}_{\mathrm{MTTF}, B, \mathrm{UPB}}\right]=\left[\widehat{T}_{\mathrm{MTTF}, B,(\zeta * B)}, \widehat{T}_{\mathrm{MTTF}, B,(B-\zeta * B)}\right],} \\ \text { BCPB } & {\left[\widehat{T}_{\mathrm{MTTF}, B, \mathrm{LPCPB}}, \widehat{T}_{\mathrm{MTTF}, B, \mathrm{UBCPB}}\right]=\left[\widehat{T}_{\mathrm{MTTF}, B,(l)}, \widehat{T}_{\mathrm{MTTF}, B,(u)}\right],}\end{cases}
$$

where $l=B \times \Phi\left(2 \Phi^{-1}\left(p^{*}\right)+\Phi^{-1}(\zeta / 2)\right), u=B \times \Phi\left(2 \Phi^{-1}\right.$ $\left.\left(p^{*}\right)+\Phi^{-1}(1-(\zeta / 2))\right)$, and $p^{*}$ is the proportion of the $B$ values $\widehat{T}_{\mathrm{MTTF}, B}$ that are less than $\widehat{T}_{\mathrm{MTTF}, M}$.
3.3. Sample Size Estimation. It is supposed that the sample size $N$ is determined by using the following condition:

$$
P\left\{(1-\varepsilon) T_{\mathrm{MTTF}} \leq \widehat{T}_{\mathrm{MTTF}, M} \leq(1+\varepsilon) T_{\mathrm{MTTF}}\right\} \geq \phi,
$$


where $\varepsilon$ is a positive constant, which can be assimilated to obtain accuracy, and satisfied $0<\varepsilon<1$, and $\phi$ which corresponds to the confidence level and is also a positive constant (see Figure 2 for a better understanding of parameters $\varepsilon$ and $\phi$ ).

Since $\widehat{T}_{\text {MTTF,M }}$ can be approximated as a normal distribution with mean $T_{\text {MTTF }}$ and variance $\operatorname{Avar}\left(\widehat{T}_{\text {MTTF, } M}\right)$, therefore, we have

$$
P\left\{-z_{(1-\phi) / 2} \leq \frac{\widehat{T}_{\mathrm{MTTF}, M}-T_{\mathrm{MTTF}}}{\sqrt{\operatorname{Avar}\left(\widehat{T}_{\mathrm{MTTF}, M}\right)}} \leq z_{(1-\phi) / 2}\right\} \geq \phi,
$$

where $z_{((1-\phi)) / 2}$ is the $((1-\phi) / 2)^{\text {th }}$ quantile of the standard normal distribution (in other words, $(1-\phi) / 2$ is the confidence level).

Comparing equations (19) and (20), the minimum sample size can be approximately determined as

$$
N^{*} \approx \frac{H^{\prime} G^{-1}(\Theta) H\left(z_{(1-\phi) / 2}\right)^{2}}{\varepsilon^{2}\left(T_{\mathrm{MTTF}}\right)^{2}},
$$

where $\quad H^{\prime}=\left(\left(\partial T_{\mathrm{MTTF}} / \partial \mu_{\alpha}\right),\left(\partial T_{\mathrm{MTTF}} / \partial \sigma_{\alpha}^{2}\right),\left(\partial T_{\mathrm{MTTF}} / \partial \beta\right)\right.$, and $\left.\left(\partial T_{\mathrm{MTTF}} / \partial \sigma_{B}^{2}\right)\right)^{\prime}$ and $G(\Theta)=(1 / N) I(\Theta)$, which is the Fisher information matrix, whose detailed expressions are listed in the Appendix.

According to (17), $H^{\prime}$ can be expressed as

$$
\left\{\begin{array}{l}
\frac{\partial T_{\mathrm{MTTF}}}{\partial \mu_{\alpha}}=\frac{2 \sqrt{2} \omega^{2}}{\sigma_{\alpha}^{3}} D\left(\frac{\mu_{\alpha}}{\sqrt{2} \sigma_{\alpha}}\right)-\frac{2 \mu_{\alpha}}{\sigma_{\alpha}^{2}} T_{\mathrm{MTTF}}, \\
\frac{\partial T_{\mathrm{MTTF}}}{\partial \sigma_{\alpha}^{2}}=\frac{\mu_{\alpha}^{2}}{\sigma_{\alpha}^{4}} T_{\mathrm{MTTF}}-\frac{1}{\sigma_{\alpha}^{2}} T_{\mathrm{MTTF}}-\frac{\sqrt{2} \mu_{\alpha} \omega^{2}}{\sigma_{\alpha}^{5}} D\left(\frac{\mu_{\alpha}}{\sqrt{2} \sigma_{\alpha}}\right), \\
\frac{\partial T_{\mathrm{MTTF}}}{\partial \beta}=\frac{\partial T_{\mathrm{MTTF}}}{\partial \sigma_{B}^{2}}=0 .
\end{array}\right.
$$

\section{Optimal Design of Accelerated Carbonation Destructive Test Plan}

Following the argument in the assumptions (see Section 2.4), for $1 \leq k \leq d, n_{k}$ indicates the number of units assigned to the stress level $s_{k}$ and $p_{k}=\left(n_{k} / N\right)$ denotes the proportion of test units that is allocated to $s_{k}$, where $\sum_{k=1}^{d} n_{k}=N$. The number of measurements is $m_{k}$ under stress level $s_{k}$. There are $n_{k j}$ units assigned to the test time $\Lambda\left(t_{k j}\right)$, such that $\sum_{j=1}^{m_{k}} n_{k j}=n_{k}$. A unit can only be tested only once, since the test is destructive. The total time on test (TTT) is $m_{k} f_{k}$, where $f_{k}$ is the inspection frequency under stress level $s_{k}$ and satisfies $f_{k}=\Lambda\left(t_{k j}\right)-\Lambda\left(t_{k(j-1)}\right)$.

In this section, we consider the optimization problem of determining the allocation of the units $\left(n_{1}, n_{2}, \ldots, n_{d}\right)$ or the proportion $\left(p_{1}, p_{2}, \ldots, p_{d}\right)$, the inspection frequency $\left(f_{k}\right)$, and the number of measurements $\left(m_{k}\right)$ according to optimization criteria under normal operating conditions subject to a prefixed budget.

\subsection{Constraints}

(1) The test time $t_{k m_{k}}, k=1, \ldots, d$ should not exceed the specified test duration $t_{A}$

(2) The sample size $\sum_{k=1}^{d} n_{k}=N$ should not exceed the number of test units available $N_{A}$

(3) The total test cost TC should not exceed the prefixed budget $C_{b}$

The total cost of conducting a CSADT can be expressed

$$
\mathrm{TC}\left(n_{1}, \ldots, n_{d}, f_{1}, \ldots, f_{d}, m_{1}, \ldots, m_{d}\right)=C_{\mathrm{op}} \sum_{k=1}^{d} f_{k} m_{k}+\left(C_{\text {mea }}+C_{d}\right) \sum_{k=1}^{d} n_{k} m_{k},
$$

where $C_{\text {op }}$ denotes the operation cost of a unit per time, $C_{\text {mea }}$ denotes the unit cost for each measurement, and $C_{d}$ denotes the unit cost for each sample.

4.2. Optimization Criteria. A-, D-, and V-optimality are three commonly used optimality criteria based on the Fisher information matrix $I(\Theta)$ (see detailed expression in Appendix). These criteria optimize the objective function from different theoretical perspectives. We will see below that A-optimality and D-optimality emphasize the estimation accuracy of the model parameters from the perspective of variance and confidence interval, respectively. V-optimality focuses on the accuracy of the estimated durability by minimizing the asymptotic variance of the estimated durability. V-optimality is most used in engineering applications where we are interested more in estimating a reliability indicator (such as MTTF, a $p$-quantile), a probability of failure, or to guarantee a performance/a lifetime with a given level of risk.

In essence, the A-, D-, and V-optimality criteria cannot be judged as good or bad. It depends on what we focus on. If we are interested to guarantee an indicator related to durability, we can choose V-optimality. If we focus on the accuracy of the model, for example, to find a maintenance strategy, we can choose D-optimality or A-optimality.

In the first simulations we will carry out, we compare the results given by the three criteria for the optimal CSADDT plans (see Section 5.2.1). But, since we focus mainly on a 


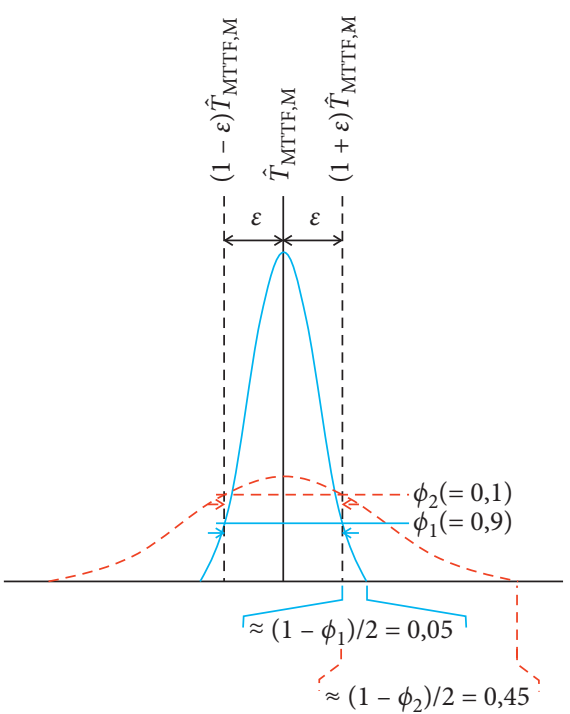

(a)

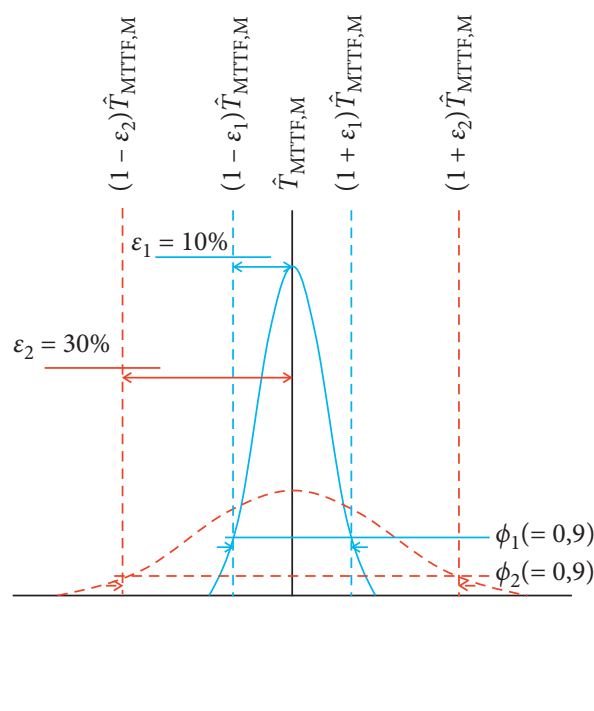

(b)

FigURE 2: Illustration of the relationship between $\widehat{T}_{\text {MTTF,M }}$, $\varepsilon$, and $\phi$ (see equation (19)). (a) Illustration of varying $\phi$ with a constant value of $\varepsilon$. (b) Illustration of varying $\varepsilon$ with a constant value of $\phi$ (values taken as $\phi_{1}=0.9$ and $\varepsilon_{1}=10 \%$, have been chosen here only for illustrative purpose).

durability indicator in our case, only V-optimality will be considered for the following simulations (from Section 5.2.2 up to the end).

Before proceeding further, we briefly review the definitions of these commonly used optimality criteria.

Criterion 1 (A-Optimality). This criterion is based on the minimization of the inverse Fisher information matrix trace. A-optimality focuses on the accuracy of parameters estimation, which is the same as the D-optimality. Unlike D-optimality, A-optimality uses the variance of parameters estimation as the accuracy. Due to the fact that the inverse of Fisher information matrix is the asymptotic variance-covariance matrix, to minimize this trace is equivalent to minimize total variance of the parameter estimates. The A-optimization problem can be formulated as follows:

$$
\begin{array}{ll}
\text { Min } & \operatorname{trace}\left(I^{-1}\left(\Theta \mid n_{1}, \ldots, n_{d}, f_{1}, \ldots, f_{d}, m_{1}, \ldots, m_{d}\right)\right), \\
\text { subject to } & \operatorname{TC}\left(n_{1}, \ldots, n_{d}, f_{1}, \ldots, f_{d}, m_{1}, \ldots, m_{d}\right) \leq C_{b}, \\
& n \leq N_{A}, t_{k, m_{k}} \leq t_{A}, \quad k=1, \ldots, d .
\end{array}
$$

Criterion 2 (D-Optimality). This second criterion is based on the maximization of the Fisher information matrix determinant. Actually, we first need to estimate the parameters of the model when we analyze the reliability of concrete. The accuracy of model parameter estimation will affect the accuracy of concrete reliability. D-optimality focuses on the accuracy of parameter estimation. The principle of this criterion is based on the fact that the overall volume of the asymptotic joint confidence region of $\Theta=\left(\mu_{\alpha}, \sigma_{\alpha}^{2}, \beta, \sigma_{B}^{2}\right)$ is proportional to $\left|I^{-1}(\Theta)\right|^{(-1 / 2)}[28,29]$. Motivated by this, maximizing the determinant of the Fisher information matrix is equivalent to minimizing asymptotic joint confidence ellipsoid of $\Theta$ and then maximizing the joint precision of the estimators of $\Theta$. The D-optimization problem can be formulated as follows:

$$
\begin{array}{ll}
\operatorname{Max} & \operatorname{det}\left(I\left(\Theta \mid n_{1}, \ldots, n_{d}, f_{1}, \ldots, f_{d}, m_{1}, \ldots, m_{d}\right)\right), \\
\text { subject to } & \operatorname{TC}\left(n_{1}, \ldots, n_{d}, f_{1}, \ldots, f_{d}, m_{1}, \ldots, m_{d}\right) \leq C_{b}, \\
& n \leq N_{A}, t_{k, m_{k}} \leq t_{A}, \quad k=1, \ldots, d .
\end{array}
$$

Criterion 3 (V-Optimality). This optimality criterion is based on the minimization of the asymptotic variance of the estimated durability at use condition. For the concrete, the durability is an important index. We need to estimate the durability at the normal or service stress level with maximum precision. Therefore, we can use the asymptotic variance of durability at normal or service stress as the optimality criterion. The asymptotic variance of durability can be obtained by using the delta method, and the formulation can be expressed as

$$
\operatorname{Avar}\left(\widehat{T}_{\text {MTTF }} \mid n_{1}, \ldots, n_{d}, f_{1}, \ldots, f_{d}, m_{1}, \ldots, m_{d}\right)=H^{\prime} I^{-1}(\Theta) H .
$$

Therefore, the V-optimization problem can be formulated as follows:

$$
\begin{array}{ll}
\text { Min } & \operatorname{Avar}\left(\widehat{T}_{\mathrm{MTTF}} \mid n_{1}, \ldots, n_{d}, f_{1}, \ldots, f_{d}, m_{1}, \ldots, m_{d}\right), \\
\text { subject to } & \operatorname{TC}\left(n_{1}, \ldots, n_{d}, f_{1}, \ldots, f_{d}, m_{1}, \ldots, m_{d}\right) \leq C_{b}, \\
& n \leq N_{A}, t_{k, m_{k}} \leq t_{A}, \quad k=1, \ldots, d .
\end{array}
$$

It is difficult to obtain the analytic expression of the optimal solution since there are random effects. Consider the feature that the variables $n_{k}, f_{k}$, and $m_{k}$ are integers. We can obtain the optimal solution after finite number of steps (Algorithm 2). 
(1) Set $n_{\max }=\min \left(\left(C_{b}-2 d C_{\mathrm{op}}\right) /\left(2 d\left(C_{\text {mea }}+C_{d}\right)\right),\left(N_{A} /(2 d)\right)\right), x$ is the floor of $x$, and $n_{\max }$ is the largest possible number for $n_{k}$ when $f_{k}=1$ and $m_{k}=2$ for $\forall k$

(2) for $n_{1}=n_{\min }: n_{\max }$ do

(3) $\ldots$

(4) for $n_{d}=n_{\min }: n_{\max }$ do

(5) Set $f_{\max }=\min \left(\left(\left(C_{b}-2\left(C_{\text {mea }}+C_{d}\right) \sum_{k=1}^{d} n_{k}\right) / 2 C_{\text {op }}\right)-d+1, t_{A} / 2\right)$, where $f_{\max }$ is the largest possible number of $f_{k}$ when $n_{k}$ is fixed and $m_{k}=2$ for $\forall k$

(6) for $f_{1}=1: f_{\max }$ do

(7) $\quad \ldots$

(8) for $f_{d}=1: f_{\max }$ do

(9) for $m_{1}=2:\left(t_{A} / f_{1}\right)$ do

Algorithm 2: The algorithm to solve the optimal solution.

The detailed algorithm for this section is given in Algorithm 2 .

\section{Application to Carbonation Degradation}

The Wiener process is used to analyze the durability of concrete. The influence of stress level, detection frequency, and number of detections on the optimal solution is discussed. We first generate the degradation path based on the physical model. And then, the parameter estimates are obtained by using the EM algorithm. Subsequently, the interval estimation can be obtained according to the bootstrap method. At last, we set the cost constraint, time constraint, and sample constraint. The optimal designs are obtained according to algorithm 2 .

5.1. Durability Estimate. The concrete used is composed of CEM I cement that can be used in prefabrication $(52.5 \mathrm{~N})$ with a water-to-equivalent binder (W/Equivalent Binder $=0.55)$, compressive strength $=35 \mathrm{MPa}$, and binder equivalent content $=300\left(\mathrm{~kg} / \mathrm{m}^{3}\right)$. All parameters necessary for the generation of data are given in Table 1.

The nominal stress $\left(\mathrm{CO}_{2}\right.$ pressure " $P_{0}$ ") corresponds to its normal concentration in the ambient air $(\sim 0.03 \%)$; thus, the lower bound of applied stress is $P_{0}=30 \mathrm{~Pa}$. The French standard for accelerated carbonation tests XP P18-458 [30] prescribes to use a concentration of $\mathrm{CO}_{2}$ equal to $50 \%$. Thus, the maximum level of applied stress will be $P_{0}=50,000 \mathrm{~Pa}$.

In the first demonstration of our generic CSADDT optimization approach, we will take two intermediary stress levels, $S_{1}=300 \mathrm{~Pa}$ and $S_{2}=20,000 \mathrm{~Pa}$, between the normal stress $(30 \mathrm{~Pa})$ and the maximum stress level $(50,000 \mathrm{~Pa})$. Using the physical model, we generate several degradation paths (for example, in our application, repeatability is satisfactory for 200 paths) at $S_{1}$ and $S_{2}$, respectively. Figure 3 shows the degradation data at two stress levels. In the actual carbonization test, the test is destructive. Therefore, we suppose that a unit can only be tested once.

A distribution analysis shows that the depth of carbonation is lognormally distributed. Thus, a prior isoprobabilistic transformation is needed to work in a normalized space of the depths of carbonation. We remind that the generic formalism of the Wiener process assumed that data are normally distributed.

Subsequently, we estimate the parameters $\Theta$ by using the EM algorithm. The estimated parameters are $\widehat{\mu}_{\alpha}=$ $0.0048358, \widehat{\sigma}_{\alpha}=0.0015086, \widehat{\beta}=1.6325$, and $\widehat{\sigma}_{B}=0.0005178$. Figure 4 shows the estimated mean degradation paths for each of the two levels used in the degradation test. From this figure, we observe that the proposed Wiener model with random effect does provide a good fit for the carbonization process. In what follows, we take the MLE of parameters, $\widehat{\theta}$, 


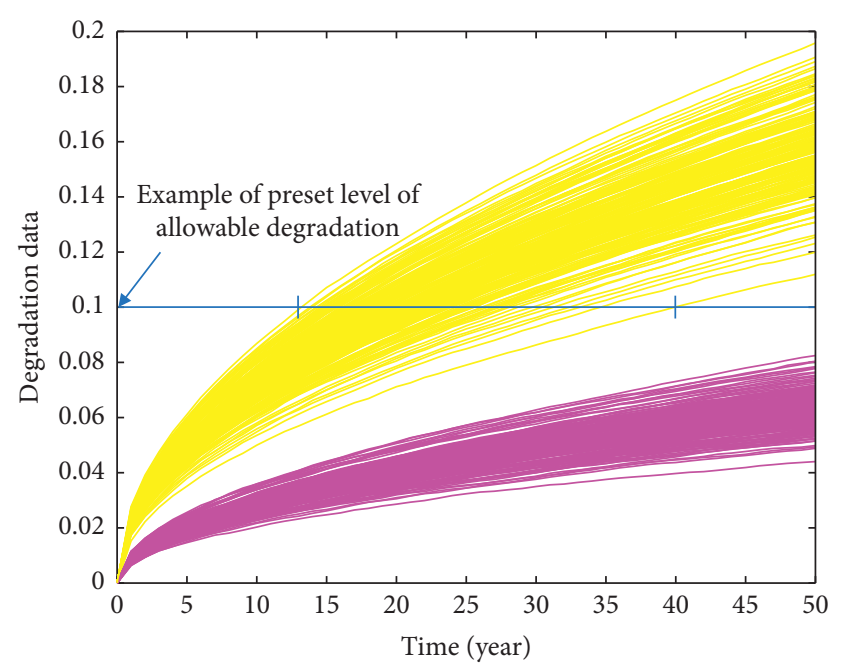

FIgURE 3: Simulated degradation data.

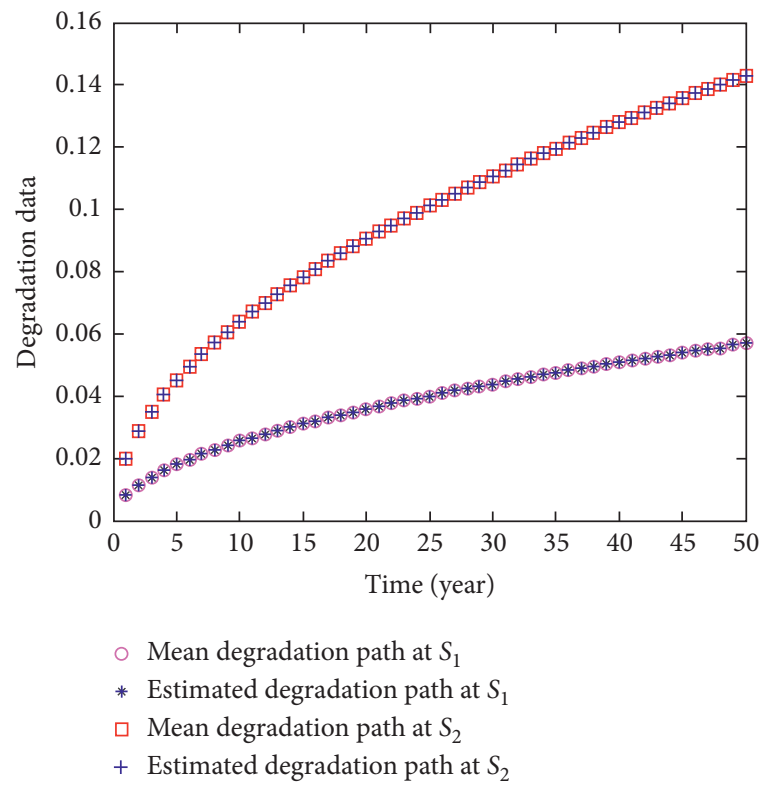

FIGURE 4: Estimated mean degradation paths.

as the true model parameters and then describe the construction of the optima design for the carbonization test.

Considering parameters $\widehat{\theta}$ and substituting threshold $w=0.035 \mathrm{~m}$ (which corresponds to a rather conventional value of concrete cover) into equation (17), we estimate an average value of mean-time-to-failure $\widehat{T}_{\text {MTTF }}$ equals to 69.5 years. A complementary interval estimation of $\widehat{T}_{\text {MTTF }}$ can be provided with a value of confidence level taken at 0.05 (i.e., $\phi=0.9)$. Using the percentile bootstrap (PB) method, we compute a confidence interval of $[59.5,81.3]$ years. The corresponding interval length is 21.8 years. By using the biaspercentile bootstrap $(\mathrm{BPB})$ method, we compute $\mathrm{CI}=[60.0$, 81.4 ] years. The corresponding interval length is 21.4 years, which is less than that from the PB method. The BPB method will be considered to have a higher accuracy than the $\mathrm{PB}$ one.

\subsection{Application of the Method on the Optimal Design of 2-Level $A D D T$}

5.2.1. Comparison of Two Scenarios. In this section, two scenarios are compared. In the first scenario denoted as $\mathrm{SI}_{1}$, $S_{1}=300 \mathrm{~Pa}$ and $S_{2}=20,000 \mathrm{~Pa}$. In the second scenario denoted as $\mathrm{SI}_{2}, S_{1}=S_{0}=30 \mathrm{~Pa}$ and $S_{2}=S_{\max }=50,000 \mathrm{~Pa}$. We will assume the following: cost factors $C_{b}=1100$ units of cost (UC), $C_{\text {mea }}=2 \mathrm{UC} /$ measurement, $C_{\text {op }}=8 \mathrm{UC} /$ unit time (here, the unit of time is 1 month, and, of course, it can be modified and expressed in days or weeks, etc.), $C_{d}=15 \mathrm{UC} /$ unit sample, $N_{A}$ is 60 samples, $t_{\max }$ is 12 months, and $n_{\min }$ is 3 samples. The costs' figures have no actual meaning, and they essentially reflect the relative importance of cost types. This minimum value of samples, at each test time under each stress level, is set at 3 so that, if one of the three measures fails, then two samples still remain to give a sense to the calculation of the averaged degradation at these test times. Table 2 sums up the optimal solutions provided following the V-optimality, D-optimality, and A-optimality conditions. According to Table 2, we have the same situation for A-optimality criteria, and for V- and $\mathrm{D}$-optimality, the total number of concrete specimens is almost the same, but the repartition is different.

Furthermore, in order to compare different optimality criteria, we calculate the corresponding confidence interval of $\widehat{T}_{\text {MTTF }}$ under the optimal scheme. The confidence level is 0.05 (i.e., $\phi=0.9$ ). The results are described in Table 3. In both scenarios $\mathrm{SI}_{1}$ and $\mathrm{SI}_{2}$, the interval length of $\widehat{T}_{\text {MTTF }}$ under $\mathrm{V}$-optimality is shorter than that of $\mathrm{D}$-optimality and A-optimality. Moreover, the length of the interval in case $\mathrm{SI}_{2}$ is shorter than the length of the interval in case $\mathrm{SI}_{1}$. These conclusions can be explained by asymptotic normality. According to asymptotic normality, the confidence interval of $T_{\text {MTTF }}$ is $\left[\widehat{T}_{\mathrm{MTTF}, M}-z_{1-(\phi / 2)} \sqrt{\operatorname{Avar}\left(\widehat{T}_{\mathrm{MTTF}, M}\right)}, \widehat{T}_{\mathrm{MTTF}, M^{+}}\right.$ $\left.z_{1-(\phi / 2)} \sqrt{\operatorname{Avar}\left(\widehat{T}_{\mathrm{MTTF}, M}\right)}\right]$. The interval width is proportional to the variance, and the $\mathrm{V}$-optimality objective is to minimize this variance. Therefore, the interval width under the $\mathrm{V}$-optimality is the smallest. Considering the absolute value of accuracy $\varepsilon$, we must admit that it is rather important. This is due to the high variability of lifetime since the degradation curves almost reach an asymptote at high value of time. We can observe it by drawing a horizontal line for an allowable preset level of degradation in Figure 1 as an illustration. This is an important point as it may suggest to try to find the sample size that satisfies both a small confidence level (high value of $\phi$ ) and a good accuracy (e.g. $\varepsilon \leq 10 \%$ ) is not a relevant strategy. For instance, for situation $\mathrm{SI}_{2}$, if we set $\phi$ to 0.95 and $\varepsilon$ to $10 \%$, the sample size should be made of about 1600 samples, which makes no sense from an industrial view.

According to Table 2 and information from the confidence interval in Table 3, we can conclude, and it makes sense, that the best optimal plan is $\mathrm{SI}_{2}$ (i.e., with $S_{1}=30 \mathrm{~Pa}$ and $\left.S_{2}=50,000 \mathrm{~Pa}\left(\mathrm{SI}_{2}\right)\right)$. Indeed, intuitively, we can understand that the more distant the two stress levels are from each other, the better the estimation of durability indicators will be (the points allowing the "regression" are distant). It will be confirmed in Section 5.2.2. 
TABLe 2: Optimal solution under different optimality criteria.

\begin{tabular}{|c|c|c|c|c|c|c|}
\hline \multirow{2}{*}{$\begin{array}{l}\text { Stress } \\
\text { Criteria }\end{array}$} & \multicolumn{3}{|c|}{$S_{1}=300 \mathrm{~Pa}, S_{2}=20,000 \mathrm{~Pa}\left(\mathrm{SI}_{1}\right)$} & \multicolumn{3}{|c|}{$S_{1}=30 \mathrm{~Pa}, S_{2}=50,000 \mathrm{~Pa}\left(\mathrm{SI}_{2}\right)$} \\
\hline & V-optimality & D-optimality & A-optimality & V-optimality & D-optimality & A-optimality \\
\hline$N_{1}\left(p_{1}\right)$ & $39(66.1 \%)$ & $30(51.7 \%)$ & $32(54.2 \%)$ & $35(59.3 \%)$ & $26(44.1 \%)$ & $32(54.2 \%)$ \\
\hline$N_{2}\left(p_{2}\right)$ & $20(33.9 \%)$ & $28(48.3 \%)$ & $27(45.8 \%)$ & $24(40.7 \%)$ & $33(55.9 \%)$ & $27(45.3 \%)$ \\
\hline Sample size & 59 & 58 & 59 & 59 & 59 & 59 \\
\hline$f_{1}$ & 1 & 1 & 1 & 1 & 1 & 1 \\
\hline$f_{2}$ & 4 & 6 & 1 & 2 & 3 & 1 \\
\hline$m_{1}$ & 3 & 2 & 8 & 7 & 2 & 8 \\
\hline$m_{2}$ & 2 & 2 & 3 & 2 & 3 & 3 \\
\hline$n_{1}$ & 13 & 15 & 4 & 5 & 13 & 4 \\
\hline$n_{2}$ & 10 & 14 & 9 & 12 & 11 & 9 \\
\hline Total test duration & 8 months & $12 \mathrm{~m}$ & $8 \mathrm{~m}$ & $7 \mathrm{~m}$ & $9 \mathrm{~m}$ & $8 \mathrm{~m}$ \\
\hline Cost & 1091 & 1098 & 1091 & 1091 & 1091 & 1091 \\
\hline
\end{tabular}

TABle 3: Comparison of different optimization criteria.

\begin{tabular}{|c|c|c|c|c|c|c|}
\hline Stress level & Optimality criteria & $\operatorname{Avar}\left(\widehat{T}_{\mathrm{MTTF}}\right)$ & $\operatorname{det}(I(\Theta))$ & $\operatorname{trace}\left(I^{-1}(\Theta)\right)$ & $\mathrm{CI}^{*}$ & Accuracy $\varepsilon(\%)$ \\
\hline \multirow{3}{*}{$S_{1}=300 \mathrm{~Pa}, S_{2}=20,000 \mathrm{~Pa}\left(\mathrm{SI}_{1}\right)$} & V-optimality & 186.8 & $5.68 e+32$ & $2.26 e-2$ & {$[42.7,96.2]$ year } & 38.6 \\
\hline & D-optimality & 198.8 & $7.67 e+32$ & $2.11 e-2$ & {$[41.8,97.1] \mathrm{y}$} & 39.8 \\
\hline & A-optimality & 201.8 & $3.46 e+32$ & $1.98 e-2$ & {$[41.6,97.3] \mathrm{y}$} & 40.1 \\
\hline \multirow{3}{*}{$S_{1}=30 \mathrm{~Pa}, S_{2}=50,000 \mathrm{~Pa}\left(\mathrm{SI}_{2}\right)$} & V-optimality & 115.7 & $9.14 e+33$ & $6.73 e-3$ & {$[48.4,90.5] \mathrm{y}$} & 30.4 \\
\hline & D-optimality & 123.4 & $1.70 e+34$ & $7.01 e-3$ & {$[47.7,91.2]$ y } & 31.3 \\
\hline & A-optimality & 115.9329 & $9.697 e+33$ & 0.00656 & {$[48.4,90.6] \mathrm{y}$} & 30.4 \\
\hline
\end{tabular}

${ }^{*}$ CI: confidence interval for $\phi=0.9$.

5.2.2. Effects of Lower and Upper Bound Values of Stress Levels. As our optimization objective is related to the minimization of the asymptotic variance of lifetime indicators and that the $\mathrm{V}$-optimality proves its better relevancy, we limit the analysis of the bound values of stress levels to this optimality criterion in the following.

In the first analysis, we study the effect of the CSADDT lower stress level. Here, the upper bound is set constant at $S_{2}=20,000 \mathrm{~Pa}$ and the lower bound $S_{1}$ is increased from 30 to $300 \mathrm{~Pa}$. When $S_{1}=30 \mathrm{~Pa}$, we are in the case of a partially CSADDT ("partially," because the lower stress level of the two levels applied corresponds to nominal or service conditions). Partially, ADDTs are often used when stress-life relationship is not known or cannot be assumed to be known, but the formalism of our model with the standardization of the stress level (i.e., standardized value of $S_{1}$ is $\left.s_{1}=s_{0}=0\right)$ is sufficiently generic to consider the test in the nominal condition as a special case of our model.

In the following calculations, the cost parameters are the same as in Section 5.2.1. In Table 4 and Figure 5, we observe that the value of $\mathrm{V}$-optimality indicator increases as the lower bound increases, and the optimal plan changes with different lower stress levels (i.e., values of $f_{i}, m_{i}$, and $n_{i}$ are different). These observations confirm those given in $\mathrm{Ta}$ bles 2 and 3 and lead us to conclude that optimality is obtained for the smaller lower bound $S_{1}=S_{\min }=30 \mathrm{~Pa}$. From a practical point of view, these results allow us to conclude that the use of a partially constant-stress ADDT should be preferred.

We complete the analysis of the bound values of stress levels by the study of the effect of CSADDT upper stress level. The lower bound is set constant at $S_{1}=300 \mathrm{~Pa}$ and the upper bound $S_{2}$ is increased from 20,000 to $50,000 \mathrm{~Pa}$. The results are summed up in Table 5 . We observe that V-optimality indicator values decrease as the upper bound increases. Compared with the last row of Table 4 (corresponding to $S_{1}=300 \mathrm{~Pa}$ ), we note that the value of the upper bound does not affect the characteristics of optimal plan $\left(f_{1}=1, f_{2}=4\right.$, $m_{1}=3, m_{2}=2, n_{1}=13$, and $\left.n_{2}=10\right)$. These results with those of Table 4 confirm that a P-CSADDT with the highest possible upper bound-the degradation mechanisms must be the same as under nominal conditions-should be chosen. Figure 6 gives a presentation of the optimal test plan.

5.2.3. Effects of Time Constraint. Results of test plan optimization above show that total test duration ranges from 6 to 8 months. But even if accelerated, these tests could be considered too long regarding industrial time constraints. In Section 5.2.3, we study the effect of the reduction of test time. This time will range from 4 to 24 weeks; the cost parameters and the sample size constraint (60 samples) are the same as initial. Specifically, we want to see if, for a shorter test time constraint, there will be a change of lower and upper bounds from the optimal values $S_{1}=30 \mathrm{~Pa}$ and $S_{2}=50,000 \mathrm{~Pa}$ found with a larger time constraint above. Table 6 sums up the results. The most significant observation is that if test time duration decreases, the optimal lower bound is no longer the nominal or service stress level as suggested for longer test duration. The partially CSADDT is no more the optimal plan. Indeed, for short test time, tests carried out at nominal conditions do not give enough information about the degradation process because this latter barely starts at low stress level for short time. This is why it seems more logical to 
TABLE 4: Solution under V-optimality for different lower bounds (upper bound $S_{2}=50,000 \mathrm{~Pa}$ ).

\begin{tabular}{lccccccccccccc}
\hline$S_{1}(\mathrm{~Pa})$ & $N_{1}\left(p_{1} \%\right)$ & $N_{2}\left(p_{2} \%\right)$ & Sample size $N$ & $f_{1}$ & $f_{2}$ & $m_{1}$ & $m_{2}$ & $n_{1}$ & $n_{2}$ & Total test duration & Cost $(\mathrm{UC})$ & Avar $\left(\widehat{T}_{\text {MTTF }}\right)$ & Accuracy $\varepsilon(\%)$ \\
\hline 30 & $35(59.3)$ & $24(40.7)$ & 59 & 1 & 2 & 7 & 2 & 5 & 12 & 7 months & 1091 & 117.2 \\
60 & $35(59.3)$ & $24(40.7)$ & 59 & 1 & 3 & 5 & 2 & 7 & 12 & 6 & 1091 & 126.5 \\
90 & $35(59.3)$ & $24(40.7)$ & 59 & 1 & 3 & 5 & 2 & 7 & 12 & 6 & 1091 & 135.2 \\
120 & $35(59.3)$ & $24(40.7)$ & 59 & 1 & 3 & 5 & 2 & 7 & 12 & 6 & 1091 & 143.5 \\
150 & $35(59.3)$ & $24(40.7)$ & 59 & 1 & 3 & 5 & 2 & 7 & 12 & 6 & 1091 & 151.5 & 33.7 \\
180 & $39(66.1)$ & $20(33.9)$ & 59 & 1 & 4 & 3 & 2 & 13 & 10 & 8 & 1091 & 158.7 \\
210 & $39(66.1)$ & $20(33.9)$ & 59 & 1 & 4 & 3 & 2 & 13 & 10 & 8 & 1091 & 165.7 & 34.7 \\
240 & $39(66.1)$ & $20(33.9)$ & 59 & 1 & 4 & 3 & 2 & 13 & 10 & 8 & 1091 & 172.8 & 36.3 \\
270 & $39(66.1)$ & $20(33.9)$ & 59 & 1 & 4 & 3 & 2 & 13 & 10 & 8 & 1091 & 179.8 \\
300 & $39(66.1)$ & $20(33.9)$ & 59 & 1 & 4 & 3 & 2 & 13 & 10 & 8 & 1091 & 186.8 & 37.8 \\
\hline
\end{tabular}

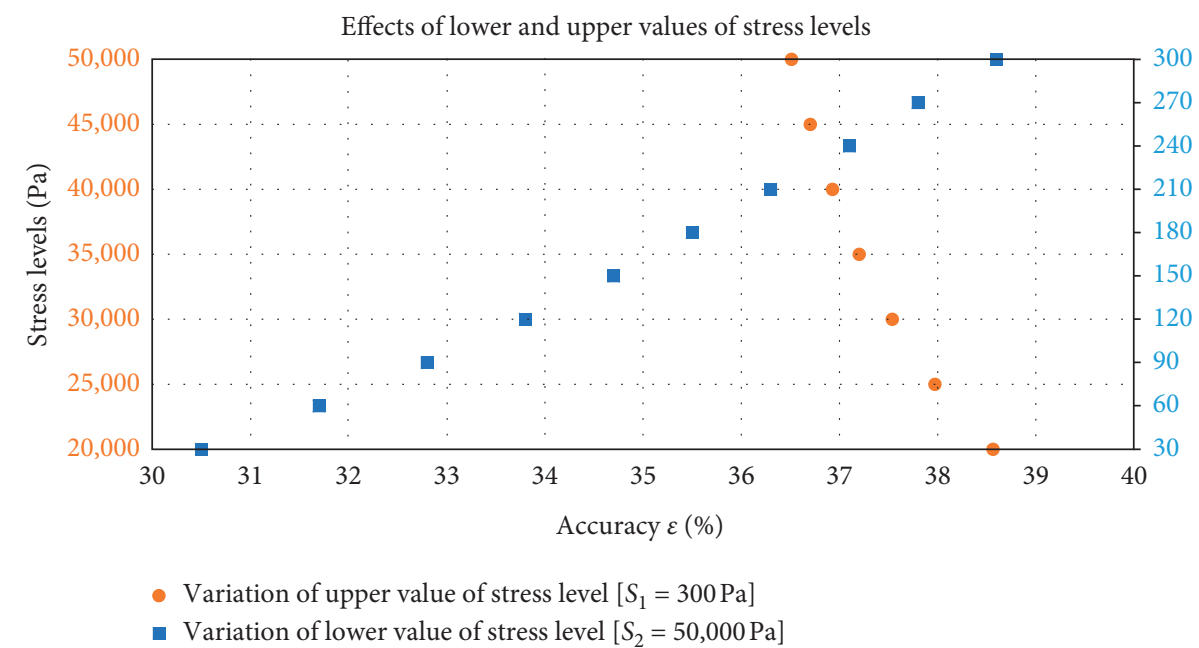

FIgURE 5: Representation of the effects of upper and lower bounds of stress levels on the estimate accuracy $\varepsilon$.

TABLE 5: Solution under V-optimality for different upper bounds (lower bound $S_{1}=300 \mathrm{~Pa}$ ).

\begin{tabular}{cccccccccccccc}
\hline$S_{2}(\mathrm{~Pa})$ & $N_{1}\left(p_{1} \%\right)$ & $N_{2}$ & Sample size $N$ & $f_{1}$ & $f_{2}$ & $m_{1}$ & $m_{2}$ & $n_{1}$ & $n_{2}$ & Total test duration & Cost (UC) & Avar $\left(\widehat{T}_{\text {MTTF }}\right)$ & Accuracy $\varepsilon(\%)$ \\
\hline 20,000 & $39(66.1)$ & $20(33.9)$ & 59 & 1 & 4 & 3 & 2 & 13 & 10 & 8 & 1091 & 186.8 \\
25,000 & $39(66.1)$ & $20(33.9)$ & 59 & 1 & 4 & 3 & 2 & 13 & 10 & 8 & 1091 & 181.1 & 38.6 \\
30,000 & $39(66.1)$ & $20(33.9)$ & 59 & 1 & 4 & 3 & 2 & 13 & 10 & 8 & 1091 & 177.02 & 38.0 \\
35,000 & $39(66.1)$ & $20(33.9)$ & 59 & 1 & 4 & 3 & 2 & 13 & 10 & 8 & 1091 & 173.9 & 37.2 \\
40,000 & $39(66.1)$ & $20(33.9)$ & 59 & 1 & 4 & 3 & 2 & 13 & 10 & 8 & 1091 & 171.3 & 36.9 \\
45,000 & $39(66.1)$ & $20(33.9)$ & 59 & 1 & 4 & 3 & 2 & 13 & 10 & 8 & 1091 & 169.3 \\
50,000 & $39(66.1)$ & $20(33.9)$ & 59 & 1 & 4 & 3 & 2 & 13 & 10 & 8 & 1091 & 167.5 & 36.7 \\
\hline
\end{tabular}

increase the low stress level in order to capture more significant information about the degradation process.

5.3. Sample Size. As shown in equation (21) and discussed at the end of Section 5.2.1, a relationship exists between the sample size (and, consequently, the constraint on the number of test units available, $N_{A}$ ), the accuracy $\varepsilon$, and the confidence level $(1-\phi) / 2$. In the present section, we want to quantify the influence of the optimality indicator and of the upper and lower bounds of stress levels on the sample size for a constant accuracy and a constant level of confidence. In other words, we relaxed the constraint on the number of tests units available $N_{A}$. We consider, as an illustration, the
$\mathrm{SI}_{1}$ scenario $\left(S_{1}=300 \mathrm{~Pa}\right.$ and $\left.S_{2}=20,000 \mathrm{~Pa}\right)$, an accuracy $\varepsilon$ of $20 \%$, and a confidence level of $0.05(\phi=0.9)$. The test duration $T_{A}$ is set here at 12 months.

From Table 7 , we see that the use of V-optimality criterion prescribes the smaller number of samples. It is logical regarding that for the same number of samples, V-optimality has shown that it gives the best accuracy (see Section 5.2.1 and Table 3). In Table 8, we show the influence of lower bound value ranging from 30 to $300 \mathrm{~Pa}$ with a constant value of $S_{2}$ set to $20,000 \mathrm{~Pa}$, for the unique case of $\mathrm{V}$-optimality. As the time constraint is high $\left(T_{A}=12\right.$ months), the partially CSADDT plan is optimal (see Section 5.2.3). In this situation, an increase in the lower bound value of stress level has a negative effect; this means that sample size increases when 


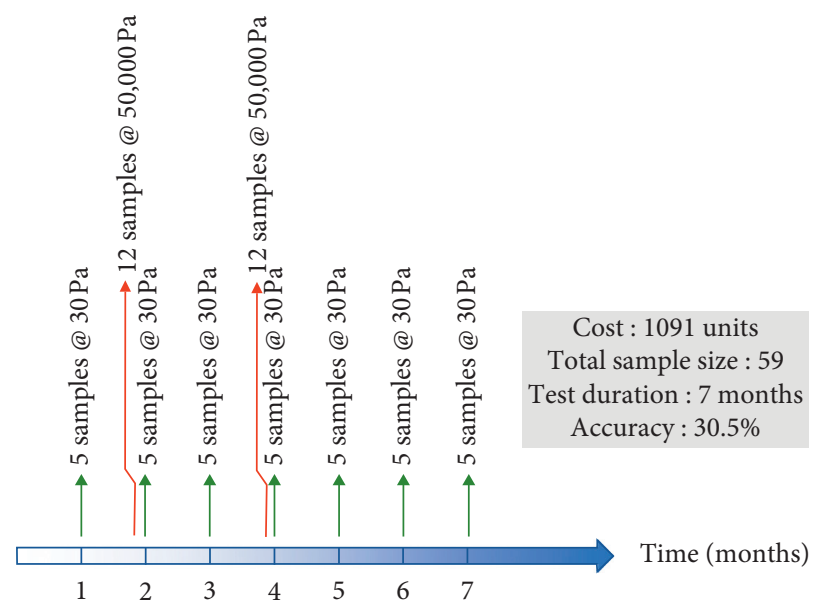

Figure 6: Timeline of the optimal two-level CSADT (with cost factors $C_{b}=1100 \mathrm{UC}, C_{\text {mea }}=2 \mathrm{UC} / \mathrm{measurement}, C_{\mathrm{op}}=8 \mathrm{UC} / \mathrm{unit}$ time, $C_{d}=15 \mathrm{UC} /$ unit sample, $N_{A}=60$ samples, $t_{\max }=12$ months, and $n_{\min }=3$ samples).

TABLE 6: Optimal bounds for varying test time constraints $t_{A}$.

\begin{tabular}{lcccccccccccc}
\hline$t_{A}$ (w.) & $f_{1}$ & $f_{2}$ & $m_{1}$ & $m_{2}$ & Total test duration & $n_{1}$ & $n_{2}$ & Sample size $N$ & $S_{1}(\mathrm{~Pa})$ & $S_{2}(\mathrm{~Pa})$ & Avar $\left(\widehat{T}_{\mathrm{MTTF}}\right)$ & Accuracy $\varepsilon(\%)$ \\
\hline 4 & 1 & 1 & 4 & 4 & 4 & 7 & 7 & 56 & 600 & 50,000 & 412.1 \\
6 & 1 & 1 & 6 & 4 & 6 & 6 & 6 & 60 & 200 & 50,000 & 401.0 & 52.9 \\
8 & 1 & 1 & 8 & 6 & 8 & 5 & 3 & 58 & 100 & 50,000 & 389.9 & 52.6 \\
12 & 1 & 2 & 12 & 6 & 12 & 4 & 2 & 60 & 60 & 50,000 & 367.7 & 52.2 \\
16 & 1 & 2 & 12 & 6 & 16 & 4 & 2 & 60 & 30 & 50,000 & 345.5 & 51.4 \\
20 & 1 & 3 & 12 & 6 & 18 & 4 & 2 & 60 & 30 & 50,000 & 323.3 & 49.3 \\
24 & 1 & 4 & 12 & 6 & 24 & 4 & 2 & 60 & 30 & 50,000 & 301.1 & 48.0 \\
\hline
\end{tabular}

TABLE 7: Sample size under three common optimality criteria.

\begin{tabular}{lcccccc}
\hline Optimality criteria & \multicolumn{2}{c}{ V-optimality } & \multicolumn{2}{c}{ D-optimality } & \multicolumn{2}{c}{ A-optimality } \\
Stress & Stress 1 & Stress 2 & Stress 1 & Stress 2 & Stress 1 & Stress 2 \\
\hline $\begin{array}{l}\text { Sample distribution }\left(N_{1}, N_{2}\right) \\
\text { Total sample size } N\end{array}$ & 102 & 53 & 85 & 80 & 89 \\
\hline
\end{tabular}

TABle 8: Sample size under different lower bounds for $S_{2}=20,000 \mathrm{~Pa}$.

\begin{tabular}{lcccccc}
\hline $\begin{array}{l}\text { Lower } \\
\text { stress }\end{array}$ & 30 & 90 & 150 & 210 & 270 & 300 \\
\hline$\left(N_{1}, N_{2}\right)$ & $(57$, & $(66$, & $(75$, & $(91$, & $(98$, & $(102$, \\
$N$ & $40)$ & $46)$ & $51)$ & $47)$ & $51)$ & $53)$ \\
$N$ & 97 & 112 & 126 & 138 & 149 & 155 \\
\hline
\end{tabular}

lower bound increases. In Table 9, we show the influence of the upper bound value ranging from 20,000 to 50,000 $\mathrm{Pa}$. We see that the higher the value of $S_{2}$ is, the lower the sample size is.

These two tables lead us to conclude (or to confirm) that for high test duration, a partially CSADDT with the higher feasible upper bound of stress level is the optimal plan.

5.4. Sensitivity Analysis. In practice, the estimated parameters $\widehat{\Theta}_{M}$ may differ from the true value $\Theta$. Therefore, it is necessary to investigate the influences of these parameters on the optimal CSADDT plan. With this aim, we compare the different $\mathrm{V}$-optimal two-stress CSADDT plans obtained under various combinations of these parameters, $\left(1+\varepsilon_{1}\right) \mu_{\alpha},\left(1+\varepsilon_{2}\right) \sigma_{\alpha}^{2},\left(1+\varepsilon_{3}\right) \beta$, and $\left(1+\varepsilon_{4}\right) \sigma_{B}^{2}$, where $\varepsilon_{1}, \varepsilon_{2}, \varepsilon_{3}$, and $\varepsilon_{4}$ denote the estimation errors for $\mu_{\alpha}, \sigma_{\alpha}^{2}, \beta$, and $\sigma_{B}^{2}$. Ten different combinations of errors are studied according to the $L_{9}\left(3^{4}\right)$ orthogonal array (see the four first columns of Table 10). The same cost factors $\left(C_{b}, C_{\text {mea }}, C_{\text {op }}, C_{d}\right)=(1100,2,8,15)$ are kept between the ten different combinations. The results are summed up in the remaining columns of Table 10 . The optimally designed values of $f_{1}, \ldots, n_{2}$ and the cost and sample size do not vary from the original design plan (with no estimation errors $\varepsilon_{i}$ ). It demonstrates the robustness of the optimized CSADDT plan and also confirms the relevancy of our approach.

\subsection{Discussion on Optimal 3-Level ADDT}

5.5.1. Comparison of 2-Level vs 3-Level Scenarios. We study in the following, once again, only the $\mathrm{V}$-optimality criterion. 
Table 9: Sample size under different upper bounds for $S_{1}=300 \mathrm{~Pa}$.

\begin{tabular}{lccccccc}
\hline Upper stress & 20,000 & 25,000 & 30,000 & 35,000 & 40,000 & 45,000 & 50,000 \\
\hline$\left(N_{1}, N_{2}\right)$ & $(102,53)$ & $(99,51)$ & $(97,50)$ & $(95,49)$ & $(94,48)$ & $(93,48)$ & $(92,47)$ \\
$N$ & 155 & 150 & 147 & 144 & 142 & 141 & 139 \\
\hline
\end{tabular}

TABLE 10: Optimal CSADDT plans under various combinations by using V-optimality.

\begin{tabular}{lcccccccccccccc}
\hline$\varepsilon_{1}(\%)$ & $\varepsilon_{2}(\%)$ & $\varepsilon_{3}(\%)$ & $\varepsilon_{4}(\%)$ & $f_{1}$ & $f_{2}$ & $m_{1}$ & $m_{2}$ & Total test duration & $n_{1}$ & $n_{2}$ & Sample size & Cost (UC) & Avar $\left(\widehat{T}_{\text {MTTF }}\right)$ & Accuracy $\varepsilon(\%)$ \\
\hline 5 & 5 & 5 & 5 & 1 & 4 & 3 & 2 & 8 & 13 & 10 & 59 & 1091 & 207.7 \\
5 & 0 & 0 & 0 & 1 & 4 & 3 & 2 & 8 & 13 & 10 & 59 & 1091 & 192.1 \\
5 & -5 & -5 & -5 & 1 & 4 & 3 & 2 & 8 & 13 & 10 & 59 & 1091 & 177.1 \\
0 & 5 & 0 & -5 & 1 & 4 & 3 & 2 & 8 & 13 & 10 & 59 & 1091 & 202.0 & 39.1 \\
0 & 0 & -5 & 5 & 1 & 4 & 3 & 2 & 8 & 13 & 10 & 59 & 1091 & 18.2 & 40.0 \\
0 & -5 & 5 & 0 & 1 & 4 & 3 & 2 & 8 & 13 & 10 & 59 & 1091 & 171.5 & 37.6 \\
-5 & 5 & -5 & 0 & 1 & 4 & 3 & 2 & 8 & 13 & 10 & 59 & 1091 & 197.3 & 39.6 \\
-5 & 0 & 5 & -5 & 1 & 4 & 3 & 2 & 8 & 13 & 10 & 59 & 1091 & 180.0 & 37.9 \\
-5 & -5 & 0 & 5 & 1 & 4 & 3 & 2 & 8 & 13 & 10 & 59 & 1091 & 167.7 & 36.6 \\
0 & 0 & 0 & 0 & 1 & 4 & 3 & 2 & 8 & 13 & 10 & 59 & 1091 & 186.8 \\
\hline
\end{tabular}

TABLE 11: Optimal solution under different two- or three-level scenarios.

\begin{tabular}{|c|c|c|c|}
\hline Stress & $\begin{array}{c}\mathrm{SI}_{1}\left(S_{1}=300 \mathrm{~Pa}\right. \\
\left.S_{2}=20,000 \mathrm{~Pa}, S_{3}=40,000 \mathrm{~Pa}\right)\end{array}$ & $\begin{array}{c}\mathrm{SI}_{2}\left(S_{1}=30 \mathrm{~Pa},\right. \\
\left.S_{2}=25,000 \mathrm{~Pa}, S_{3}=50,000 \mathrm{~Pa}\right)\end{array}$ & $\begin{array}{c}\mathrm{SI}_{3}\left(S_{1}=30 \mathrm{~Pa}, S_{2}=\varnothing,\right. \\
\left.S_{3}=50,000 \mathrm{~Pa}\right)\end{array}$ \\
\hline$N_{1}\left(p_{1}\right)$ & $35(59.3 \%)$ & $35(59.3 \%)$ & $39(66.1 \%)$ \\
\hline$N_{2}\left(p_{2}\right)$ & $6(10.2 \%)$ & $6(10.2 \%)$ & $\varnothing$ \\
\hline$N_{3}\left(p_{3}\right)$ & $18(30.5 \%)$ & $18(30.5 \%)$ & $20(33.9 \%)$ \\
\hline$f_{1}$ & 1 & 1 & 1 \\
\hline$f_{2}$ & 1 & 1 & $\varnothing$ \\
\hline$f_{3}$ & 2 & 1 & 4 \\
\hline$m_{1}$ & 5 & 7 & 3 \\
\hline$m_{2}$ & 2 & 2 & $\varnothing$ \\
\hline$m_{3}$ & 2 & 3 & 2 \\
\hline Total duration & 5 months & 7 months & 8 months \\
\hline$n_{1}$ & 7 & 5 & 13 \\
\hline$n_{2}$ & 3 & 3 & $\varnothing$ \\
\hline$n_{3}$ & 9 & 6 & 10 \\
\hline Sample size & 59 & 59 & 59 \\
\hline Cost & 1091 & 1099 & 1091 \\
\hline $\operatorname{Avar}\left(\widehat{T}_{\text {MTTF }}\right)$ & 98.9 & 58.1 & 115.7 \\
\hline Accuracy $\varepsilon(\%)$ & 28.2 & 22.4 & 30.4 \\
\hline
\end{tabular}

We compare three scenarios: $\mathrm{SI}_{1} \quad\left(S_{1}=300 \mathrm{~Pa}\right.$, $\left.S_{2}=20,000 \mathrm{~Pa}, \quad S_{3}=40,000 \mathrm{~Pa}\right) ; \quad \mathrm{SI}_{2} \quad\left(S_{1}=30 \mathrm{~Pa}\right.$, $\left.S_{2}=25,000 \mathrm{~Pa}, S_{3}=50,000 \mathrm{~Pa}\right) ; \mathrm{SI}_{3} \quad\left(S_{1}=30 \mathrm{~Pa}, S_{2}=\varnothing\right.$, $\left.S_{3}=50,000 \mathrm{~Pa}\right)$. The third scenario is the reference twolevel P-CSADDT. We suppose that the cost factors $C_{b}=1100 \mathrm{UC}, C_{\text {mea }}=2 \mathrm{UC} /$ measurement, $C_{\text {op }}=8 \mathrm{UC} /$ unit time, and $C_{d}=15 \mathrm{UC} /$ unit sample, the unit time is 1 month, $N_{A}$ is 60 samples, $t_{A}$ is 12 months, and $n_{\min }$ is 3 samples. From Table 11, it is obvious that a three-level scenario is better than a two-level one and that the larger the range between lower and upper bounds is, the better the accuracy is. As an illustration, between the two-level scenario $\mathrm{SI}_{3}$ and the best three-level scenario $\mathrm{SI}_{2}$, the accuracy is improved by about $36 \%$. It may also be noted that the test duration is also slightly reduced for the threelevel scenarios. But a question remains about the choice of the optimal value of the intermediary value of stress. It is addressed in the next section.
5.5.2. Research of Optimal Intermediate Stress Level for a 3Level CSADDT Plan. Here, the lower bound and the upper bound are kept constant and set at $S_{1}=S_{\min }=30 \mathrm{~Pa}$ and $S_{3}=S_{\max }=50,000 \mathrm{~Pa}$, respectively. The intermediate stress level $S_{2}=S_{\text {int }}$ increases from $30 \mathrm{~Pa}$ to $50,000 \mathrm{~Pa}$. The optimal solutions are summed up in Table 12. Only the values of $\operatorname{Avar}\left(\widehat{T}_{\text {MTTF }}\right)$ and of accuracy $\varepsilon$ are shown here. Indeed, all the characteristics of the optimal plans $\left(f_{1}\right.$, etc., cost) remain constant with a changing value of $S_{2}$. Figure 7 completes this analysis. We see that an optimal value of intermediary stress level can be found. This optimal value is around 10,000 Pa; it provides an accuracy of about 19\%, which is improved by about $60 \%$ (and, with the same cost, sample size and test duration) and $18 \%$ compared, respectively, to initial scenarios $\mathrm{SI}_{3}$ (2-level P-CSADDT) and $\mathrm{SI}_{2}$ (3-level P-CSADDT with the intermediary stress level equidistant to the lower and upper bounds). Figure 8 gives the timeline of the best 3 level P-CSADDT. 
TABLE 12: Optimal solution under V-optimality for different intermediate stress levels.

\begin{tabular}{lcccccccccccc}
\hline$S_{\text {int }}(\mathrm{Pa})$ & 30 & 2500 & 5000 & 10,000 & 15,000 & 20,000 & 25,000 & 30,000 & 35,000 & 40,000 & 45,000 & 50,000 \\
\hline Avar $\left(\widehat{T}_{\text {MTTF }}\right)$ & 115.7 & 69.1 & 49.5 & 35.8 & 37.6 & 43.5 & 58.1 & 74.6 & 86.0 & 99.3 & 109.6 & 115.7 \\
Accuracy $\varepsilon(\%)$ & 30.4 & 24.0 & 21.1 & 19.0 & 19.3 & 20.2 & 22.4 & 24.8 & 26.5 & 28.2 & 29.3 & 30.4 \\
\hline
\end{tabular}

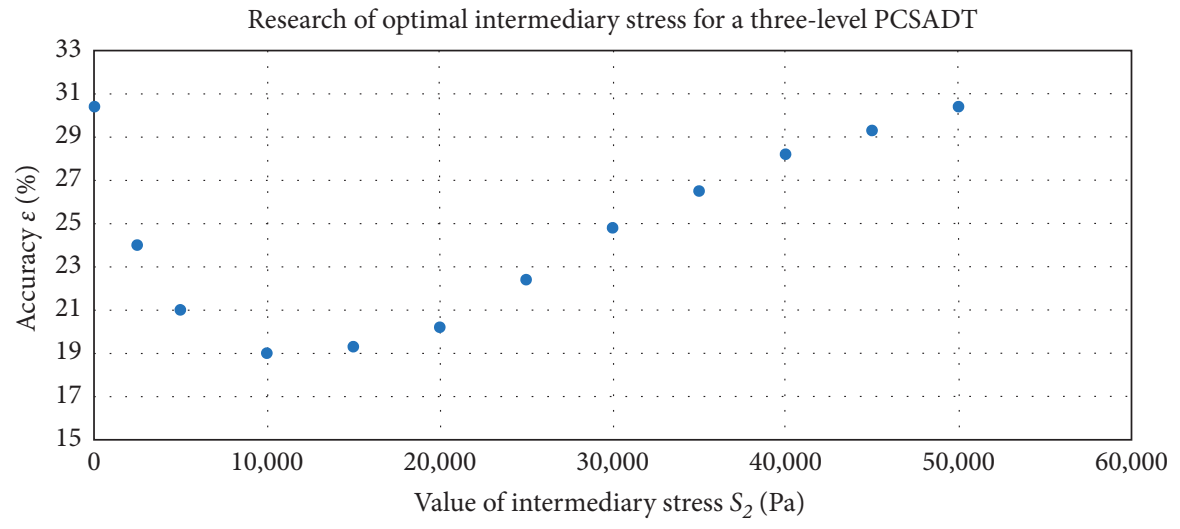

Figure 7: Representation of the effects of intermediary stress on the estimate accuracy $\varepsilon$ for a three-level P-CSADT.

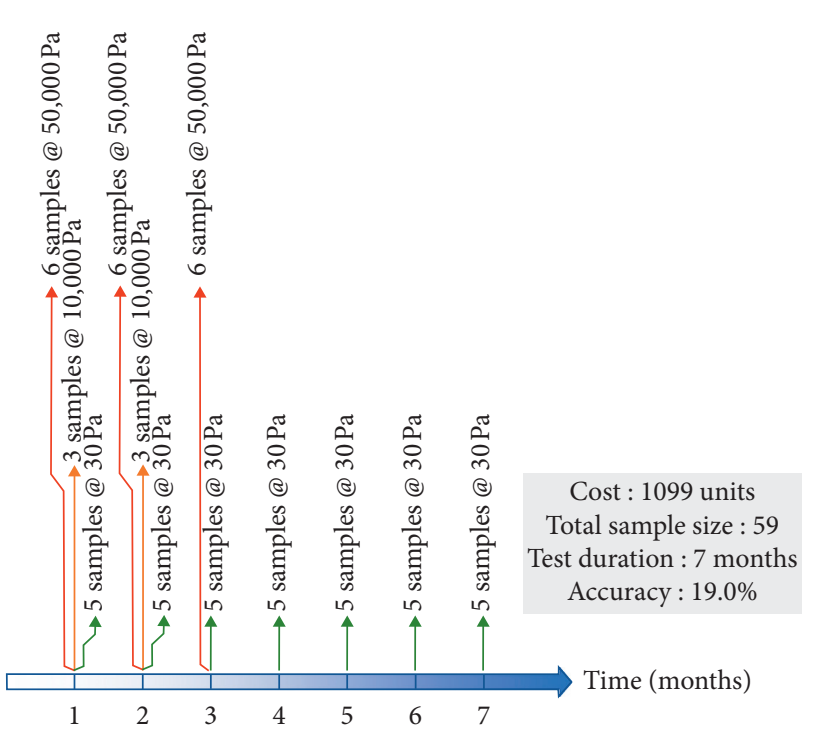

FIgURE 8: Timeline of the optimal three-level CSADT (with cost factors $C_{b}=1100 \mathrm{UC}, C_{\text {mea }}=2 \mathrm{UC} /$ measurement, $C_{\text {op }}=8 \mathrm{UC} /$ unit time, $C_{d}=15 \mathrm{UC} /$ unit sample, $N_{A}=60$ samples, $t_{\max }=12$ months, and $n_{\min }=3$ samples).

\section{Conclusion}

A generic approach, based on a nonlinear generalized Wiener process, has been developed with the aim to design constant-stress optimal accelerated carbonation tests and to estimate the durability of concrete. Here, the acceleration stress for carbonation is the $\mathrm{CO}_{2}$ pressure at the concrete surface, which can range from $30 \mathrm{~Pa}$ (the nominal or service value) to $50,000 \mathrm{~Pa}$ (the highest value prescribed in French standard for accelerated carbonation tests [30]).

The stochastic Wiener process has been chosen to model the carbonation depth evolution which is assimilated as a degradation process. Even if the Wiener process is not formally dedicated to monotonic degradation, we argue on its ability to take into account the measurement errors and the variability between samples. Due to the high sensitivity of the Wiener process formalism and the optimization issues arising therefrom, the parameters of the process have been estimated with the maximum likelihood method completed with the two-step Expectation-Maximization one. Once the parameters are determined, it is possible to design the optimal test plan, providing the best accuracy in the estimation of durability with the lower cost, sample size, or test time. Among three optimality criteria (A-, D-, and V-optimality), we recommend to choose the $\mathrm{V}$ - criterion since we seek here to estimate a durability indicator related to a desired level of guarantee durability (through the MTTF or a $p$-quantile of lifetime) and since $\mathrm{V}$-optimality shows a direct relationship between durability indicators. On this basis, we have looked for the optimal configuration for a two-level constant-stress accelerated degradation test (CSADDT).

We have shown the relevancy of partially (where the lower stress level is taken at the nominal or service level) constantstress accelerated degradation tests (P-CSADDT) in the case of long test duration. But, for reduced test times, the choice of P-CSADDT is not so intuitive and we have shown that alternatives with higher lower bounds are optimal.

In the last part of the paper, we have investigated how a three-level CSADDT would improve the estimation of durability. After showing that an optimal value for the third intermediary stress level exists, we have underlined that an improvement of $60 \%$ of the estimate accuracy is possible with the same cost, same sample size, and same test duration.

To conclude, we think that this generic approach can be very useful for qualification of new concrete mixes by means of optimized accelerated tests. This approach can be applied to concrete subjected to other environments (for example, environment with chlorides or freeze and thaw). 


\section{Appendix}

\section{A1. Expression of Fisher Information Matrix}

The expression of Fisher information matrix $I(\Theta)$ is

$$
I(\Theta)=\left[\begin{array}{llll}
E\left(-\frac{\partial^{2} l}{\partial\left(\mu_{\alpha}\right)^{2}}\right) & E\left(-\frac{\partial^{2} l}{\partial \mu_{\alpha} \partial \sigma_{\alpha}^{2}}\right) & E\left(-\frac{\partial^{2} l}{\partial \mu_{\alpha} \partial \beta}\right) & E\left(-\frac{\partial^{2} l}{\partial \mu_{\alpha} \partial \sigma_{B}^{2}}\right) \\
E\left(-\frac{\partial^{2} l}{\partial \sigma_{\alpha}^{2} \partial \mu_{\alpha}}\right) & E\left(-\frac{\partial^{2} l}{\partial\left(\sigma_{\alpha}^{2}\right)^{2}}\right) & E\left(-\frac{\partial^{2} l}{\partial \sigma_{\alpha}^{2} \partial \beta}\right) & E\left(-\frac{\partial^{2} l}{\partial \sigma_{\alpha}^{2} \partial \sigma_{B}^{2}}\right) \\
E\left(-\frac{\partial^{2} l}{\partial \beta \partial \mu_{\alpha}}\right) & E\left(-\frac{\partial^{2} l}{\partial \beta \partial \sigma_{\alpha}^{2}}\right) & E\left(-\frac{\partial^{2} l}{\partial \beta^{2}}\right) & E\left(-\frac{\partial^{2} l}{\partial \beta \partial \sigma_{B}^{2}}\right) \\
E\left(-\frac{\partial^{2} l}{\partial \sigma_{B}^{2} \partial \mu_{\alpha}}\right) & E\left(-\frac{\partial^{2} l}{\partial \sigma_{B}^{2} \partial \sigma_{\alpha}^{2}}\right) & E\left(-\frac{\partial^{2} l}{\partial \sigma_{B}^{2} \partial \beta}\right) & E\left(-\frac{\partial^{2} l}{\partial\left(\sigma_{B}^{2}\right)^{2}}\right)
\end{array}\right],
$$

where $E\left(-\left(\partial^{2} l / \partial\left(\mu_{\alpha}\right)^{2}\right)\right)=N \sum_{k=1}^{d} \sum_{j}^{m_{k}}\left(p_{k j} e^{2 \beta s_{k}} \tau_{k j}\right) / B_{1 k j}, E$ $\left(-\left(\left(\partial^{2} l\right) /\left(\partial \mu_{\alpha} \partial \sigma_{\alpha}^{2}\right)\right)\right)=0, \quad E\left(-\left(\left(\partial^{2} l\right) /\left(\partial \mu_{\alpha} \partial \sigma_{B}^{2}\right)\right)\right)=0, \quad E(-$ $\left.\left(\left(\partial^{2} l\right) /\left(\partial \mu_{\alpha} \partial \beta\right)\right)\right)=N \sum_{k=1}^{d} \sum_{j}^{m_{k}}\left(p_{k j} \mu_{\alpha} s_{k} e^{2 \beta s_{k}} \tau_{k j}\right) /\left(B_{1 k j}\right), E(-$ $\left.\left(\left(\partial^{2} l\right) /\left(\partial\left(\sigma_{\alpha}^{2}\right)^{2}\right)\right)\right)=N \sum_{k=1}^{d} \sum_{j}^{m_{k}}\left(p_{k j} / 2\right)\left(\left(e^{2 \beta s_{k}} \tau_{k j}\right) /\left(B_{1 k j}\right)\right)^{2}, E$ $\left(-\left(\partial^{2} l /\left(\partial \sigma_{\alpha}^{2} \partial \beta\right)\right)\right)=N \sum_{k=1}^{d} \sum_{j}^{m_{k}}\left(p_{k j} s_{k} \sigma_{\alpha}^{2} e^{4 \beta s_{k}} \tau_{k j}^{2}\right) /\left(B_{1 k j}^{2}\right), E(-$ $\left.\partial^{2} l / \partial \sigma_{\alpha}^{2} \partial \sigma_{B}^{2}\right)=N \sum_{k=1}^{d} \sum_{j}^{m_{k}} p_{k j} e^{2 \beta s_{k}} \tau_{k j} / 2 B_{1 k j}^{2}, \quad E\left(-\left(\left(\partial^{2} l\right) /\right.\right.$ $\left.\left.\left(\partial \beta^{2}\right)\right)\right)=N \sum_{k=1}^{d} \sum_{j}^{m_{k}} p_{k j}\left(2 s_{k}^{2}+\left(\left(2 s_{k}^{2} \sigma_{B}^{4}\right) /\left(B_{1 k j}^{2}\right)\right)+\left(\left(s_{k}^{2} \mu_{\alpha}^{2} e^{2 \beta s_{k}}\right.\right.\right.$ $\left.\left.\left.\tau_{k j}\right) /\left(B_{1 k j}\right)\right)-\left(\left(4 s_{k}^{2} \sigma_{B}^{2}\right) /\left(B_{1 k j}\right)\right)\right), E\left(-\left(\partial^{2} l / \partial \beta \partial \sigma_{B}^{2}\right)\right)=N \sum_{k=1}^{d}$ $\sum_{j}^{m_{k}}\left(p_{k j} s_{k} \sigma_{\alpha}^{2} e^{2 \beta s_{k}} \tau_{k j}\right) / B_{1 k j}^{2}$, and $E\left(-\left(\left(\partial^{2} l\right) /\left(\partial\left(\sigma_{B}^{2}\right)^{2}\right)\right)\right)=$ $N \sum_{k=1}^{d} \sum_{j}^{m_{k}}\left(p_{k j} /\left(2 B_{1 k j}^{2}\right)\right)$.

\section{A2. Expression of $\mathbf{p}$-Quantile}

Conditional on $\alpha$, the degradation $Y_{\text {mod }}(t \mid \alpha)$ follows a normal distribution with mean $\alpha e^{\beta s_{k}} \Lambda(t)$ and variance $\sigma_{B}^{2} \Lambda(t)$. Marginalizing over $\alpha$, the unconditional distribution of $Y_{\bmod }(t)$ follows normal distribution with mean $\mu_{\alpha} e^{\beta s_{k}} \Lambda(t)$ and variance $\sigma_{B}^{2} \Lambda(t)+$ $\sigma_{B}^{2} \Lambda^{2}(t)$. Therefore, the CDF of $T$ can be approximated as

$$
F_{T}(t)=\Phi\left(\frac{\mu_{\alpha} e^{\beta s_{k}} \Lambda(t)-\omega}{\sqrt{\sigma_{B}^{2} \Lambda(t)+\sigma_{B}^{2} \Lambda^{2}(t)}}\right) .
$$

Denote the standard normal quantile $\Phi^{-1}(p)$ as $z_{p}$. Inverting (A.2), an approximation of the $p$-th lifetime quantile is

$$
\xi_{p}=\left(\frac{2 \omega \mu_{\alpha}+z_{p}^{2} \sigma_{B}^{2}+z_{p} \sqrt{z_{p}^{2} \sigma_{B}^{4}+4 \omega \mu_{\alpha} \sigma_{B}^{2}+4 \omega^{2} \sigma_{\alpha}^{2}}}{2\left(\mu_{\alpha}^{2}-z_{p}^{2} \sigma_{\alpha}^{2}\right)}\right)^{2} .
$$

\section{Data Availability}

The data used to support the findings of this study are included within the article. Most of them are deduced from models described in the article.

\section{Conflicts of Interest}

The authors declare that they have no conflicts of interest.

\section{Acknowledgments}

The authors would like to acknowledge great support by the ANRT (National Association of Research and Technology, France), ATILH (Technical Association of the Hydraulic Binder Industry, France), and National Natural Science Foundation of China (71861011).

\section{References}

[1] EN 206 2014, Performance, Production and Conformity, Concrete-Specification, 2014.

[2] J. A. Bickley, R. D. Hooton, and K. C Hover, "Performance specifications for durable concrete," Concrete International, vol. 28, pp. 51-57, 2006.

[3] E. G. Abdelouafi, K. Benaissa, and K. Abdellatif, "Reliability analysis of reinforced concrete buildings: comparison between FORM and ISM," Procedia Engineering, vol. 114, pp. 650-657, 2015.

[4] I. Allahyari, Concrete Performance Approach: Towards a Better Characterization of Sustainability Indicators, Paul SabatierToulouse University III, Toulouse, France, 2016.

[5] M. Hanifehzadeh, M. Ebad Sichani, B. Gencturk, and J. E. Padgett, "Destructive and non-destructive evaluation of reinforced concrete dry casks affected by alkali-silica reactivity damage," Structure and Infrastructure Engineering, vol. 15, no. 10, pp. 1404-1418, 2019.

[6] L. Capozzoli and E. Rizzo, "Combined NDT techniques in civil engineering applications: laboratory and real test," Construction and Building Materials-Virtual Special Issue Ground-Penetrating Radar and Complementary Non-Destructive Testing Techniques in Civil Engineering, vol. 154, pp. 1139-1150, 2017.

[7] Z. Yu, Z. Ren, J. Tao, and X. Chen, “Accelerated testing with multiple failure modes under several temperature conditions," Mathematical Problems in Engineering, vol. 2014, Article ID 839042, 8 pages, 2014. 
[8] W. Q. Meeker, G. Sarakakis, and A. Gerokostopoulos, "More pitfalls of accelerated tests," Journal of Quality Technology, vol. 45, no. 3, pp. 213-222, 2017.

[9] F. Duan and G. Wang, "Optimal design for constant-stress accelerated degradation test based on gamma process," Communications in Statistics-Theory and Methods, vol. 48, no. 9, pp. 2229-2253, 2019.

[10] C.-C. Tsai, S.-T. Tseng, and N. Balakrishnan, “Optimal design for degradation tests based on gamma processes with random effects," IEEE Transactions on Reliability, vol. 61, no. 2, pp. 604-613, 2012.

[11] X. Liu, Z. Wu, D. Cui, B. Guo, and L. Zhang, "A modeling method of stochastic parameters' inverse gauss process considering measurement error under accelerated degradation test," Mathematical Problems in Engineering, vol. 2019, p. 11, 2014.

[12] S. Tang, X. Guo, C. Yu, H. Xue, and Z. Zhou, “Accelerated degradation tests modeling based on the nonlinear wiener process with random effects," Mathematical Problems in Engineering, vol. 2014, Article ID 560726, 11 pages, 2014.

[13] Z. Chen, S. Li, and E. Pan, "Optimal constant-stress accelerated degradation test plans using nonlinear generalized wiener process," Mathematical Problems in Engineering, vol. 2016, Article ID 9283295, 11 pages, 2016.

[14] L. Sun, X. Gu, and P. Song, "Accelerated degradation process analysis based on the nonlinear wiener process with covariates and random effects," Mathematical Problems in Engineering, vol. 2016, Article ID 5246108, 13 pages, 2016.

[15] N. Hyvert, Application of the Probabilistic Approach to the Sustainability of Prefabricated Concrete Products, Paul Sabatier-Toulouse III University, Toulouse, France, 2009.

[16] C. J. Lu and W. O. Meeker, "Using degradation measures to estimate a time-to-failure distribution," Technometrics, vol. 35, no. 2, pp. 161-174, 1993.

[17] L. I. Pettit and K. D. S. Young, "Bayesian analysis for inverse Gaussian lifetime data with measures of degradation," Journal of Statistical Computation and Simulation, vol. 63, no. 3, pp. 217-234, 1999.

[18] X. Wang, P. Jiang, B. Guo, and Z. Cheng, "Real-time reliability evaluation with a general wiener process-based degradation model," Quality and Reliability Engineering International, vol. 30, no. 2, pp. 205-220, 2014.

[19] H. Qiao, B. Zhu, C. Lu, Q. Feng, M. Zhou, and H. Cao, "Accelerated life test of concrete based on wiener stochastic process," Journal of Building Materials and Structures, vol. 19, pp. 1023-1027, 2016.

[20] K. Zhang and J. Xiao, "Time-dependent reliability analysis on carbonation behavior of recycled aggregate concrete based on gamma process," Construction and Building Materials, vol. 158, pp. 378-388, 2018.

[21] R. Neves, F. A. Branco, and J. de Brito, "A method for the use of accelerated carbonation tests in durability design," Construction and Building Materials, vol. 36, pp. 585-591, 2012.

[22] A. V. Saetta, B. A. Schrefler, and R. V. Vitaliani, "The carbonation of concrete and the mechanism of moisture, heat and carbon dioxide flow through porous materials," Cement and Concrete Research, vol. 23, no. 4, pp. 761-772, 1993.

[23] A. Morandeau, M. Thiéry, and P. Dangla, "Investigation of the carbonation mechanism of $\mathrm{CH}$ and $\mathrm{C}-\mathrm{S}-\mathrm{H}$ in terms of kinetics, microstructure changes and moisture properties," Cement and Concrete Research, vol. 56, pp. 153-170, 2014.

[24] H. Lim and B.-J. Yum, "Optimal design of accelerated degradation tests based on Wiener process models," Journal of Applied Statistics, vol. 38, no. 2, pp. 309-325, 2011.
[25] M. S. Chang, Y. I. Kwon, and B. S. Kang, "Design of reliability qualification test for pneumatic cylinders based on performance degradation data," Journal of Mechanical Science and Technology, vol. 28, no. 12, pp. 4939-4945, 2014.

[26] G. J. Mc Lachlan and T. Krishnan, The EM Algorithm and Extensions, Wiley, Hoboken, NJ, USA, 2nd edition, 2008

[27] Q. Guan and Y. C. Tang, "Optimal design of accelerated degradation test based on gamma process models," Chinese Journal of Applied Probability and Statistics, vol. 29, pp. 213-224, 2013.

[28] X. Wang, "Wiener processes with random effects for degradation data," Journal of Multivariate Analysis, vol. 101, no. 2, pp. 340-351, 2010.

[29] C.-H. Hu, M.-Y. Lee, and J. Tang, "Optimum step-stress accelerated degradation test for Wiener degradation process under constraints," European Journal of Operational Research, vol. 241, no. 2, pp. 412-421, 2015.

[30] XP P 18-458 2008, Tests for Hardened Concrete-Accelerated Carbonation Test-Measurement of the Thickness of Carbonated Concrete 2008. 